\title{
Influence of higher order modes on the beam stability in the high power superconducting proton linac
}

\author{
Marcel Schuh* \\ Beams Department, CERN, Geneva, Switzerland, and MPI-K, Heidelberg, Germany \\ Frank Gerigk and Joachim Tückmantel \\ Beams Department, CERN, Geneva, Switzerland \\ Carsten P. Welsch \\ The University of Liverpool and Cockcroft Institute, Warrington, United Kingdom
}

(Received 3 November 2010; published 4 May 2011)

\begin{abstract}
Higher order modes (HOMs) can severely limit the operation of superconducting cavities in a linear accelerator with high beam current, high duty factor, and complex pulse structure. The full HOM spectrum has to be analyzed in order to identify potentially dangerous modes already during the design phase and to define their damping requirements. For this purpose a dedicated beam simulation code simulation of higher order mode dynamics (SMD) focused on beam-HOM interaction was developed, taking into account important effects like the HOM frequency spread, beam input jitter, different chopping patterns, as well as klystron and alignment errors. Here, SMD is used to investigate the influence of HOMs in detail in the superconducting proton linac at CERN and their potential to drive beam instabilities in the longitudinal and transverse plane.
\end{abstract}

DOI: 10.1103/PhysRevSTAB.14.051001

PACS numbers: 29.27.Bd, 29.20.Ej

\section{INTRODUCTION}

The superconducting proton linac (SPL) [1-3] is conceived as a $4 \mathrm{MW}$ high power superconducting linac in pulsed operation, as driver for a neutrino or a radioactive ion beam facility. It accelerates $H^{-}$from $160 \mathrm{MeV}$ up to $5 \mathrm{GeV}$ using two families $\left(\beta_{g}=0.65\right.$ and $\beta_{g}=1.0$ ) of five cell superconducting elliptical $\pi$-mode cavities, operating at $704.4 \mathrm{MHz}$. A pulse length of $\leq 1 \mathrm{~ms}$ is foreseen with an average pulse current up to $40 \mathrm{~mA}$ and a repetition rate of $50 \mathrm{~Hz}$. All basic design parameters of the linac and the cavities used in this study are listed in Tables I and II.

The linac consists of two sections with different cavities. In the medium beta section, nine periods are planned, each containing six elliptical $\beta_{g}=0.65$ five cell cavities and two quadrupole doublets, see Fig. 1. Afterwards 24 periods with eight elliptical $\beta_{g}=1.0$ five cell cavities and one quadrupole doublet compose the high beta section, see Fig. 2. There are also alternative layouts with other sectorizations proposed [4], which entail only minor differences with respect to beam dynamics and which are not discussed in this paper. Possible

\footnotetext{
*Marcel.Schuh@cern.ch

Published by the American Physical Society under the terms of the Creative Commons Attribution 3.0 License. Further distribution of this work must maintain attribution to the author(s) and the published article's title, journal citation, and DOI.
}

extraction regions along the linac are not considered in this study.

Beam instabilities caused by higher order modes (HOMs) are of concern in high power superconducting linacs and have already been treated in various publications [5-8]. The experience gained at SNS [9,10] raises the question, if dedicated HOM couplers are really needed in machines like SPL, ESS [11,12], or Project X [13,14].

In this paper the code simulation of higher order mode dynamics (SMD) [15] is introduced, which is developed to simulate the HOM-beam interaction in the longitudinal

TABLE I. SPL parameters used in this study.

\begin{tabular}{lcc}
\hline \hline Parameter & & Value \\
\hline Output energy & {$[\mathrm{GeV}]$} & 5 \\
Beam power & {$[\mathrm{MW}]$} & $>4.0$ \\
Repetition rate & {$[\mathrm{Hz}]$} & 50 \\
Average pulse current & {$[\mathrm{mA}]$} & 40 \\
Peak pulse current & {$[\mathrm{mA}]$} & 64 \\
Beam pulse length & {$[\mathrm{ms}]$} & 1.0 \\
Bunch frequency & {$[\mathrm{MHz}]$} & 352.2 \\
Number of sections & & 2 \\
Number of cavities & & $54 / 192$ \\
Cavities/cryostat & & $6 / 8$ \\
Period length & {$[\mathrm{m}]$} & $12.25 / 15.06$ \\
Maximum power/cavity & {$[\mathrm{MW}]$} & 1 \\
Length & {$[\mathrm{m}]$} & 472 \\
\hline \hline
\end{tabular}


TABLE II. Cavity design parameters.

\begin{tabular}{lccc}
\hline \hline Parameter & & Medium $\beta$ & High $\beta$ \\
\hline Cells & & 5 & 5 \\
Frequency & {$[\mathrm{MHz}]$} & 704.4 & 704.4 \\
Geometrical beta $\beta_{g}$ & & 0.65 & 1.0 \\
$(R / Q)\left(\beta_{g}\right)$ & {$[\Omega]$} & 320 & 560 \\
Design gradient $E_{0} T\left(\beta_{g}\right)$ & {$[\mathrm{MV} / \mathrm{m}]$} & 19 & 25 \\
$Q_{0}$ & & $5.8 \times 10^{9}$ & $8.4 \times 10^{9}$ \\
\hline \hline
\end{tabular}

and transverse plane. A special focus is set on the implementation of the longitudinal dynamics of $\beta<1$ beams. SMD is benchmarked against existing codes such as, for example, TALOBBU [7] to verify its performance.

The influence of various parameters is analyzed, such as mean HOM frequency, HOM frequency spread, substructured pulses, cavity alignment, and injection noise as a function of beam current and HOM damping separately for the longitudinal and transverse plane. In the longitudinal plane the impact of rf errors is studied to define tolerable limits for HOM induced beam degradation.

Based on the simulation results, HOM damping requirements for different operation modes will be defined.

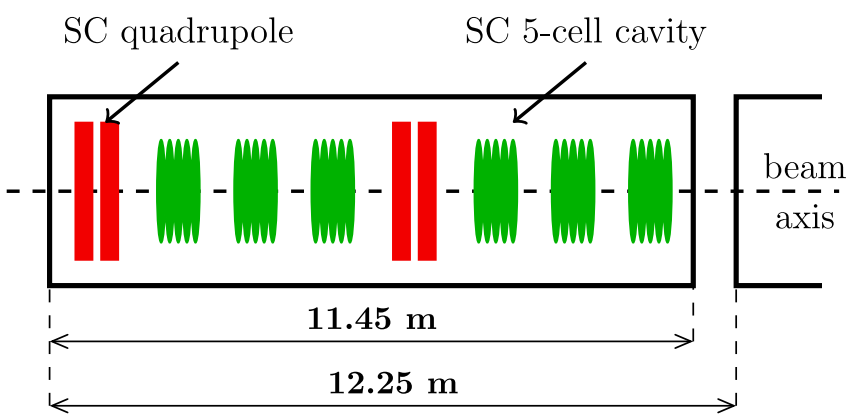

FIG. 1. Period layout in the medium beta section.

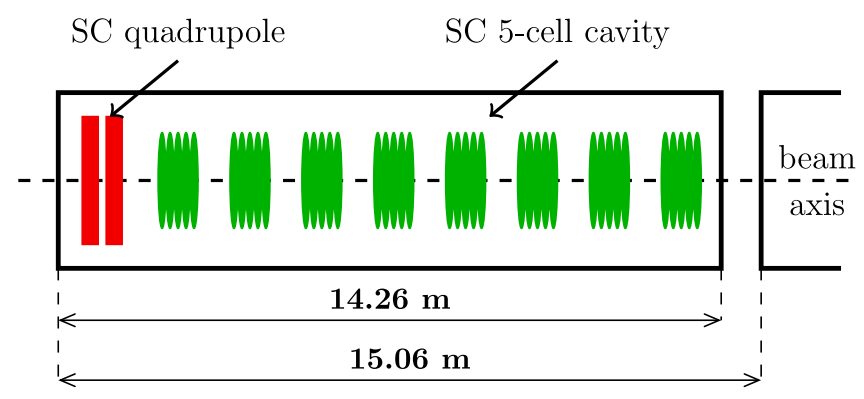

FIG. 2. Period layout in the high beta section.

\section{BEAM PHYSICS}

In this section a brief summary is given of the basics of higher order mode excitation and beam interaction based on [8,16-18]. For simplification, a complex notation for the voltage is used. By convention, the observable physical quantities are real and the sign of the imaginary phase is positive $[\exp (+i \omega t)]$. Compared to the considered HOM wavelength, the bunch length in the SPL is very short [19], meaning that bunches can be treated as pointlike charges. The coordinate system is chosen such that the beam always travels along the $z$ axis (beam axis) in positive direction. The electrical $z$ axis in the cavity is assumed to be identical with the geometrical $z$ axis.

A point charge traveling along the beam axis excites all monopole modes inside a cavity. Since all modes are orthogonal to each other, they can be discussed separately. The mode specific voltage induced by a point charge $q$ is

$$
\Delta V_{q, n}=-q \frac{\omega_{n}}{2}(R / Q)_{n}(\beta),
$$

where $\omega_{n}$ is the angular frequency of the mode and $\beta$ is the relativistic factor of the particle velocity. $(R / Q)_{n}(\beta)$ of a particular mode with an angular frequency $\omega_{n}$ takes into account the velocity-dependent transit time factor and is defined [20] as

$$
(R / Q)_{n}(\beta)=\frac{\left|\int_{-\infty}^{\infty} E_{n, z}(r=0, z) \exp \left(i \omega_{n} \frac{z}{\beta c}\right) d z\right|^{2}}{\omega_{n} U_{n}},
$$

where $E_{n, z}$ is the field component along the beam axis and $U_{n}$ the stored energy of mode $n$. The integration borders are set to infinity to consider also the fields leaking out of the cavity into the beam pipe.

A bunch passes a cavity, where no fields are present beforehand, and induces the voltage $\Delta V_{q, n}$ in mode $n$, which starts oscillating at $t=0$, when the bunch passes the cavity midplane [21]. Because of losses the voltage decays over time according to

$$
V_{n}(t)=\Delta V_{q, n} \exp \left(-t / T_{d, n}\right) \exp \left(i \omega_{n} t\right),
$$

where the decay time constant $T_{d, n}$ is

$$
T_{d, n}=\frac{2 Q_{L, n}}{\omega_{n}} .
$$

$Q_{L, n}$ is the quality factor of the complete system for this mode. It is the inverse sum of all subsystems

$$
\frac{1}{Q_{L, n}}=\frac{1}{Q_{\mathrm{ex}, n}}+\frac{1}{Q_{0, n}},
$$

where $Q_{0, n}$ is the value for the unloaded cavity mode and $Q_{\text {ex }, n}$ is the total quality factor of the external loads, which are the fundamental power coupler, HOM coupler, and dampers such as bellows. The natural $Q_{0}$ of a superconducting cavity is in the order of $10^{10}$. So $Q_{L}$ is dominated by $Q_{\mathrm{ex}}$ and in this paper the simplification $Q_{L} \approx Q_{\mathrm{ex}}$ is 
made. $Q_{\mathrm{ex}, n}$ depends on the coupling efficiency to the particular mode and can vary over several orders of magnitude from mode to mode. So-called trapped modes have most of their stored energy in the center cells, which means that they do not or only very weakly couple to HOM couplers or the power coupler. In consequence trapped modes have generally a very high $Q_{\mathrm{ex}}$. In this paper $Q_{\mathrm{ex}}$ is used as a simulation parameter and is not calculated from EM simulations for the particular modes.

The voltage induced by the next bunch arriving after the time $T_{b}$ in the same mode is added as vector sum to the voltage already present in the cavity. Under the assumption that the time between bunches is constant and that there is no charge scatter, the equilibrium HOM voltage in continuous wave $(\mathrm{CW})$ operation can be derived by evaluating the sum of the induced voltages directly:

$$
\begin{aligned}
V_{\mathrm{cw}, n} & =\Delta V_{q, n} \sum_{k=0}^{\infty} \exp \left(-k T_{b} / T_{d}+i k \omega_{n} T_{b}\right) \\
& =\frac{\Delta V_{q, n}}{1-\exp \left(-T_{b} / T_{d}+i \omega_{n} T_{b}\right)} .
\end{aligned}
$$

From this formula, one can easily see that a HOM with $f=k / T_{b}, k \in \mathbb{N}$ will be excited on resonance, which are the principal machine lines.

In pulsed operation the voltage after one pulse with $N$ bunches and no HOM voltage present before the first bunch arrives is then

$$
\begin{aligned}
V_{p, n} & =\Delta V_{q, n} \sum_{k=1}^{N} \exp \left(-k T_{b} / T_{d}+i k \omega_{n} T_{b}\right) \\
& =\Delta V_{q, n} \frac{1-\exp \left(-N T_{b} / T_{d}+i N \omega_{n} T_{b}\right)}{1-\exp \left(-T_{b} / T_{d}+i \omega_{n} T_{b}\right)} .
\end{aligned}
$$

If there are substructures in the pulse train, the formula can be extended to

$$
V_{p, n}=\Delta V_{q, n} \prod_{j=0}^{M} \frac{1-\exp \left(-k_{j} T_{j} / T_{d}+i k_{j} \omega_{n} T_{j}\right)}{1-\exp \left(-T_{j} / T_{d}+i \omega_{n} T_{j}\right)},
$$

where $k_{j}$ is the number of repetitions, $T_{j}$ the period length of the substructure, and $M$ the number of structure elements. $M=0$ corresponds to $\mathrm{CW}$ operation, $M=1$ to pulsed operation, and $M>1$ to pulsed operation with further substructures.

Because of the chopping of the regular bunch sequence, additional weaker chopping machine lines appear at

$$
f_{\mathrm{c}}=k / T_{j}, \quad k \in \mathbb{N},
$$

that can also excite a HOM on resonance. These resonances, created by the pulse substructure, are called chopping machine lines in the further discussion. The smaller $T_{j}$, the stronger is the resonance, which can be explained by looking at the Fourier components of the excitation.
Hence, the most dangerous resonances are the principal machine lines, created by the bunch spacing $T_{b}$.

Dipole modes can only be excited by off-axis particles, because they do not have any field component $\left(E_{z}\right)$ along the beam axis. The following convention is used for dipole fields in this paper: $E_{z}(x, y, z)=E_{z}(r, z) \cos (\phi)$ with $x=r \cos (\phi)$ and $y=r \sin (\phi)$. The polarization axis is chosen such that the maximum electric field is along the $x$ axis and so $\phi=0 . E_{z}$ rises approximately linearly with the transverse coordinate $x$ as long as $x$ is smaller than the cavity iris radius $R_{\text {iris. }}$. A deviation from the $x$ axis can be neglected in a first order approximation because $\partial E_{z} / \partial y=0$.

The particles get deflected in the field of a dipole mode inside a cavity. Using the Panofski-Wenzel theorem [22,23] this can be expressed as

$$
\Delta p_{\perp, n}=i \frac{q}{\omega_{n}} \frac{d V_{z, n}}{d x},
$$

where $\omega_{n}$ is the angular frequency of the mode $n$. A socalled "transverse voltage" $V_{\perp, n}$ can be defined and linked to the voltage $V_{\|, n}(x, \beta)$ induced by a charge $q$ traveling with the speed $\beta c$ parallel to the beam axis at distance $x$ :

$$
V_{\perp, n}=\frac{c p_{\perp}}{q}=i \frac{c}{\omega_{n} x} V_{\|, n}(x, \beta),
$$

while using the fact that for dipole modes the longitudinal voltage scales linearly with $x$. Similar to the longitudinal $(R / Q)_{n}(\beta)$ the same parameter can be introduced for dipole modes,

$$
(R / Q)_{\perp, n}(\beta)=\frac{\left|V_{\perp, n}(\beta)\right|^{2}}{\omega_{n} U_{n}}
$$

with an integration path parallel to the beam axis at distance $x_{0}$ using relation (11):

$$
V_{\perp, n}=\frac{i c}{\omega_{n} x_{0}} \int_{-\infty}^{\infty} E_{n, z}\left(r=x_{0}, z\right) \exp \left(i \omega_{n} \frac{z}{\beta c}\right) d z
$$

A pointlike bunch of charge $q$, passing the cavity off axis with distance $x$, induces in a dipole mode with an angular frequency $\omega_{n}$ a purely imaginary transverse voltage given by

$$
\Delta V_{\perp}=\frac{1}{2} i x q \frac{\omega_{n}^{2}}{c}(R / Q)_{\perp, n}(\beta) .
$$

The energy conservation is discussed in Appendix B.

If a pulse, consisting of several bunches, passes a cavity, one has to sum up the induced voltages taking into account their phase relationship and their exponential decay. Concerning the induced longitudinal voltages, Eqs. (6)-(8) are also valid for dipole modes under the assumption that $x$ is constant.

These formulas can be used for first estimates of the expected HOM voltage in resonant excitation. Away from the machine lines, destructive interference occurs. In a real 
machine bunch noise, such as arrival time errors, energy errors, transverse displacement and tilt, and charge jitter, has to be considered in this context, leading to a much higher voltage as one would expect from assuming a perfect beam off resonance. This can even drive instabilities as shown in [8]. That is why numerical studies have to be performed to estimate the effect of HOMs.

\section{A. Longitudinal beam dynamics}

The charge to mass ratio is the same for a single particle and a bunch of particles of the same kind. So all following equations are also valid for a pointlike bunch of particles. In contrast to electron linacs, particles enter a hadron linac with a speed far below the speed of light. In this case the change of the particle velocity along the linac has various consequences, such as, for example, a velocity-dependent time of flight, which has to be included in the simulation.

An energy error leads to a transit time factor change from cavity to cavity and additionally a change of the maximum accelerating voltage $V_{\text {rf }}$ in a cavity. This, together with the phase error of the particle, leads to a different energy gain in the cavity as compared to the synchronous particle. Because of errors in the rf system, an additional phase and amplitude error of the accelerating mode has to be considered. The error in the energy gain per cavity is then

$$
d U_{\mathrm{rf}}=q V_{\mathrm{rf}}^{*} \cdot \cos \left(\phi_{s}^{*}+\omega_{\mathrm{rf}} d t\right)-\Delta U,
$$

where $V_{\mathrm{rf}}^{*}$ is the maximum accelerating voltage including transit time factor correction and amplitude errors. $\phi_{s}^{*}$ is the effective operation phase $\left(\phi_{s}^{*}=\phi_{s}+\phi_{\mathrm{rf} \text { error }}\right)$ of the cavity and $\omega_{r} d t$ is the arrival phase error of the particle. $\Delta U$ is the energy gain of the synchronous particle without rf errors,

$$
\Delta U=q V_{0} \cos \left(\phi_{s}\right)=q E_{0} T\left(\beta_{s}\right) l \cos \left(\phi_{s}\right) .
$$

A charged particle passing the cavity is subject to an additional energy change caused by an excited HOM,

$$
d U_{n}=q\left[\Re\left(V_{n}\right) \cos \left(\omega_{n} d t\right)-\Im\left(V_{n}\right) \sin \left(\omega_{n} d t\right)\right]-\frac{1}{2} \Delta V_{q, n},
$$

where $\Re\left(V_{n}\right)$ and $\Im\left(V_{n}\right)$ are the real and imaginary part of the HOM voltage, present at the transit of the cavity's midplane. In addition, half of the HOM voltage induced by the particle itself acts back on it, which is known as the fundamental theorem of beam loading [24]. Summing up all these effects leads to a total energy error evolution in cavity $m$ of

$$
d E^{(m+1)}=d E^{(m)}+d U_{\mathrm{rf}}^{(m)}+d U_{n}^{(m)},
$$

where $d E^{(m)}$ is the energy error in front of the cavity $m$. This energy error $d E^{(m+1)}$ translates into an arrival time error when passing through the drift space between cavity $m$ and $m+1$ :

$$
d t^{(m+1)}=d t^{(m)}+(d t / d E)_{E}^{(m)} \cdot d E^{(m+1)} .
$$

The slope depends on the drift length $L^{(m)}$, the mass $m_{0}$ and relativistic $\gamma_{s}$ of the synchronous particle,

$$
(d t / d E)_{E}^{m}=-\frac{L^{(m)}}{c \cdot m_{0} c^{2} \cdot\left(\gamma_{s}^{2}-1\right)^{3 / 2}} .
$$

The longitudinal beam dynamics along a linac can be fully described by Eqs. (18) and (19).

\section{B. Transverse beam dynamics}

The particles get deflected in the field of a dipole mode inside a cavity, where the deflection is, to very good approximation, independent of the transverse position $(x, y)$, where the particle passes the cavity. According to the Panofski-Wenzel theorem [22,23], a complex voltage $V_{\perp}$ yields a transverse momentum kick,

$$
\Delta p_{x}=q \frac{\mathfrak{R}\left(V_{\perp}\right)}{c},
$$

which gives rise to a change of the transverse trajectory inclination for small angles of

$$
\Delta x^{\prime}=\Delta p_{x} / p_{\|}=q \frac{\mathfrak{R}\left(V_{\perp}\right)}{c p_{\|}},
$$

where $p_{\|}$is the longitudinal momentum and $\Re\left(V_{\perp}\right)$ the real part of the HOM voltage. There is no direct interaction between the voltage induced by a particle and the particle itself, since there is a phase difference of $90^{\circ}$ between them for dipole modes.

The transverse focusing is done in the SPL by one quadrupole doublet per focusing period. This can be simplified by assuming a constant phase advance per focusing period and translating this into a smooth focusing between the cavities. The transfer matrix between two cavities with focusing is then given by

$$
\left(\begin{array}{c}
x_{n+1} \\
x_{n+1}^{\prime}
\end{array}\right)=\left(\begin{array}{cc}
\cos (L / \beta) & \beta \sin (L / \beta) \\
-\frac{\sin (L / \beta)}{\beta} & \cos (L / \beta)
\end{array}\right) \cdot\left(\begin{array}{c}
x_{n} \\
x_{n}^{\prime}
\end{array}\right),
$$

where $L$ is the distance between the cavities and $\beta$ the beam optics beta function, considered locally/piecewise constant. If there are no deflecting HOMs, the particle oscillates with a wavelength of $2 \pi \beta$ in the transverse plane.

\section{SIMULATION CODE}

A linac containing different sections and periodic substructures with a given cavity spacing is modeled using a drift-kick approach. Pointlike bunches are tracked through the complete linac. The particle velocity is assumed constant inside the cavity and the interaction between particle and cavity takes place in the cavity midplane. In [8] the implementation of the beam-HOM interaction used in SMD is described in detail. It is assumed that there is no 
coupling between the longitudinal and the transverse plane so they can be treated independently. Space charge effects are neglected in all simulations.

SMD is implemented in ROOT [25] to allow for a fast, compiled program core and using the framework routines for data analysis. The execution time for simulating two pulses consisting of 350000 single bunches is about $10 \mathrm{~s}$ in the transverse and $45 \mathrm{~s}$ in the longitudinal plane on a desktop PC with a Intel ${ }^{\circledR}$ Core $^{\mathrm{TM}} 2$ Duo E6750 with 4 GB RAM. Only one core is used in the simulation and about 250 MB RAM. The simulation code has two logical modules: One for the longitudinal plane and one for the transverse plane.

\section{A. Longitudinal module}

Field maps of the $\mathrm{TM}_{010, \pi}$ mode in the different cavities are used to derive the $E_{0} T(\beta)$ for the synchronous particle in each cavity. A two point linear interpolation is used for all other particles to model explicitly the deviation in the acceleration kick of nonsynchronous particles caused by the energy error, which translates into a different transit time factor [26]. The fields in the cavities are normalized to the design $E_{0} T\left(\beta_{g}\right)$, taking into account a certain phase advance limitation per period and the design phase $\phi_{s}$. Furthermore, amplitude and phase errors can be applied individually to each cavity.

A bunch is defined as lost, if the absolute phase $\phi$ of the bunch is outside the limits

$$
2 \phi_{s}<\phi<-\phi_{s}
$$

where $\phi_{s}$ is design phase, which is negative using the linac convention. Longitudinal beam dynamics simulations can be carried out to study effects of input errors in terms of energy and phase errors, as well as cavity gradient or rf errors.

In addition, one HOM with individual characteristic values per cavity is applied, which can be excited by a pulsed beam. It is also possible to preserve the induced voltage or set it to a certain value in order to perform studies with a fixed HOM voltage. The action back on the beam can also be controlled individually for each cavity.

\section{B. Transverse module}

The transverse plane is modeled assuming smooth focusing in the intercavity drift space using $2 \times 2$ transfer matrices. For simplicity no explicit focusing lattices are modeled. Instead the transverse focusing profile of multiparticle simulations is used in the transfer matrices.

In the initial transverse beam dynamics model the following basic assumptions and simplifications are made: (i) no alignment errors in the focusing elements; (ii) cavities have no transverse displacement; (iii) the beam is injected on axis with a normal distributed bunch to bunch spread in position and transverse momentum; (iv) one dipole mode is considered per cavity with its maximum field in the simulated plane; (v) the beam is longitudinally matched, there are no HOM monopoles; (vi) all errors are Gaussian distributed.

A simplified model of transversely displaced cavities is implemented to study alignment issues in the context of dipole modes. For this purpose a Gaussian distributed transverse position offset is used in all cavities. This affects only the HOM excitation, but not the transverse focusing. A bunch is defined as lost, if the transverse displacement is larger than a defined threshold value.

\section{Modeled effects}

In the modules a variety of effects is implemented. They can be divided into machine and beam caused effects. The modeled machine caused effects are the impact of different HOM parameters such as frequency, frequency spread, $(R / Q)_{n}(\beta)$, and $Q_{\mathrm{ex}}$. Each cavity has its own set of independent parameters. Second, rf errors and cavity gradient errors can be applied individually to each cavity. This opens a wide range of scenarios which can be investigated. In the case of beam based effects, the influence of the basic beam parameters such as current and different pulse substructures can be studied, as well as beam injection noise in terms of energy, phase, position, momentum, and bunch charge jitter. For noise generation independent Mersenne Twistor random number generators [27] are used for each quantity.

\section{Benchmark}

In order to verify the obtained results, a benchmark in the longitudinal plane against other codes $[7,8]$ was performed. 50000 bunches were tracked through a generic linac and the output was compared. The results agree perfectly within the numerical errors [28]. A detailed discussion can be found in [29].

\section{SIMULATION INPUT PARAMETERS}

In order to run beam dynamics simulations, cavity HOM data and beam input parameters are needed. They are introduced in this section. All parameters are based on results from the SPL HOM workshop [30] held at CERN in 2009. Linac4 [31,32], presently under construction at CERN, will be used as injector front end for all simulations.

\section{A. Cavity modeling}

Since the final cavity geometries are not yet confirmed, preliminary symmetrical cavity shapes are used for this study. The medium beta cavity is based on [33] and the high beta cavity is a rescaled TESLA cavity [34-36]. The exact geometries are listed in Appendix A. A HOM analy-

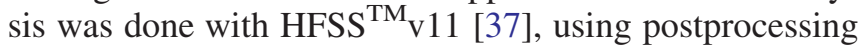
scripts [38]. The TM monopole [39] and dipole modes with 
TABLE III. Monopole modes with the highest $(R / Q)_{n}(\beta)$ values found in the symmetrical SPL cavity shapes. The $\mathrm{TM}_{010}$ modes are discussed separately in Sec. V J.

\begin{tabular}{lcc|lcr}
\hline \hline & \multicolumn{3}{c|}{$\beta_{g}=0.65$} & \multicolumn{3}{c}{$\beta_{g}=1.0$} \\
Mode & $f_{n}[\mathrm{MHz}]$ & $(R / Q)_{\text {max }, n}{ }^{\mathrm{a}}[\Omega]^{\mathrm{b}}$ & \multicolumn{1}{c}{ Mode } & $f_{n}[\mathrm{MHz}]$ & $(R / Q)_{\max , n}{ }^{\mathrm{a}}[\Omega]^{\mathrm{b}}$ \\
\hline $\mathrm{TM}_{020}, 1 / 5 \pi$ & 1505 & 6 & $\mathrm{TM}_{011}, 4 / 5 \pi$ & 1322 & 39 \\
$\mathrm{TM}_{020}, 2 / 5 \pi$ & 1511 & 12 & $\mathrm{TM}_{011}, \pi$ & 1331 & 140 \\
$\mathrm{TM}_{011}, 3 / 5 \pi$ & 1773 & 6 & $\mathrm{TM}_{021}$ & 2087 & 10 \\
$\mathrm{TM}_{011}, 4 / 5 \pi$ & 1780 & 15 & $\mathrm{TM}_{021}$ & 2090 & 21 \\
$\mathrm{TM}_{011}, \pi$ & 1783 & 44 & $\mathrm{TM}_{022}, \pi$ & 2449 & 9 \\
\hline $\mathrm{TM}_{01}$ cutoff & 2550 & & $\mathrm{TM}_{01}$ cutoff & 1639 \\
\hline \hline
\end{tabular}

${ }^{a}$ Maximum value in covered velocity range.

${ }^{\mathrm{b}}$ Linac definition.

TABLE IV. Dipole modes with the highest $(R / Q)_{\perp}(\beta)$ values found in the symmetrical SPL cavity shapes.

\begin{tabular}{|c|c|c|c|c|c|}
\hline & $\beta_{g}=1$ & & & $\beta_{g}=$ & \\
\hline Mode & $f_{n}[\mathrm{MHz}]$ & $(R / Q)_{\perp, \max , n}{ }^{\mathrm{a}}[\Omega]^{\mathrm{b}}$ & Mode & $f_{n}[\stackrel{\mathrm{MHz}}{\mathrm{M}}]$ & $(R / Q)_{\perp, \max , n}{ }^{\mathrm{a}}[\Omega]^{\mathrm{b}}$ \\
\hline $\mathrm{TM}_{110}, 1 / 5 \pi$ & 1015 & 143 & $\mathrm{TE}_{111}, 3 / 5 \pi$ & 915 & 57 \\
\hline $\mathrm{TM}_{110}, 2 / 5 \pi$ & 1020 & 107 & $\mathrm{TE}_{111}, 4 / 5 \pi$ & 940 & 60 \\
\hline $\mathrm{TM}_{111}, 3 / 5 \pi$ & 1027 & 57 & $\mathrm{TM}_{110}$ & 1014 & 36 \\
\hline $\mathrm{TM}_{110}, 4 / 5 \pi$ & 1033 & 30 & $\mathrm{TM}_{110}$ & 1020 & 25 \\
\hline $\mathrm{TE}_{111}, 1 / 5 \pi$ & 1270 & 34 & Hybrid & 1409 & 20 \\
\hline $\mathrm{TE}_{11}$ cutoff & 1952 & & $\mathrm{TE}_{11}$ cutoff & 1255 & \\
\hline
\end{tabular}

${ }^{\mathrm{a}}$ Maximum value in covered velocity range.

${ }^{\mathrm{b}}$ Linac definition.

the highest $(R / Q)_{n}$ values, found for both cavity families in the considered velocity range, are listed in Tables III and IV. The dipole modes classified as TE modes do also have TM components since pure TE modes does not exist in elliptical cavities due to the openings. This longitudinal $E$-field component is essential to excite the mode. All listed monopole modes are also analyzed at $\beta_{g}$ with SUPERFISH [40] for comparison. The change of $(R / Q)_{n}$ as a function of the beam velocity is shown in Fig. 3. Especially in the medium beta cavity no mode with a constantly high $(R / Q)_{n}$ value can be observed.

The HOM with the highest overall $(R / Q)_{n}(\beta)$ below the beam pipe cutoff frequency in each linac section is chosen as default for all simulations. A Gaussian HOM frequency spread of $\sigma_{f_{\text {ном }}}=1 \mathrm{MHz}$ is assumed for all modes based on the experience at DESY [41] and Jefferson Laboratory [42].

In this paper the focus is set to modes below the beam pipe cutoff frequency with artificially high $(R / Q)_{n}$ values. However, there are also potentially dangerous modes above the cutoff frequency. Their frequency and $(R / Q)_{n}(\beta)$ values depend strongly on the intercavity section and the cavity-to-cavity coupling. Hence, the complete cryomodule has to be simulated as it is done in [43]. With these simulations, trapped modes can be identified and the cavity and/or cryomodule design will then have to be adapted if necessary. The influence of a mode with moderate $(R / Q)_{n}(\beta) \sim 1$, weak damping, and resonant excitation on the beam will be discussed in Sec. VG 4 .

Introducing mechanical imperfections, the $(R / Q)_{n}$ and $Q_{\text {ex }}$ of a HOM in a cavity can change significantly, as shown in [44]. This effect has to be taken into account when specifying the HOM damping requirements. One can try to quantify the effect by studying the influence of mechanical tolerances on $(R / Q)_{n}(\beta)$ and $Q_{\mathrm{ex}}$. Instead of performing a statistical study we carry out simulations with very conservative assumptions and large safety margins (e.g. on the beam current) to compensate for this effect.

\section{B. Beam input parameters}

Simulation values based on Linac4 beam dynamics studies [45] are used as beam input parameters. The relevant values for the bunch energy and phase jitter in the longitudinal plane as well as the position and momentum spread in the transverse plane are listed together with all other beam parameters in Table V. In the default simulation settings, a $1 \mathrm{~ms}$ pulse with no substructure is used and the beam current is set to $\left\langle I_{b}\right\rangle=400 \mathrm{~mA}$, which corresponds to 10 times the nominal current, as a safety margin.

Beside the position/momentum or the energy/phase jitter, a bunch to bunch charge jitter of $\sigma_{q}=3 \%$ is used as a very conservative estimation [46]. All input noise is 
Monopole modes $\beta_{\mathrm{g}}=\mathbf{0 . 6 5}$ cavity

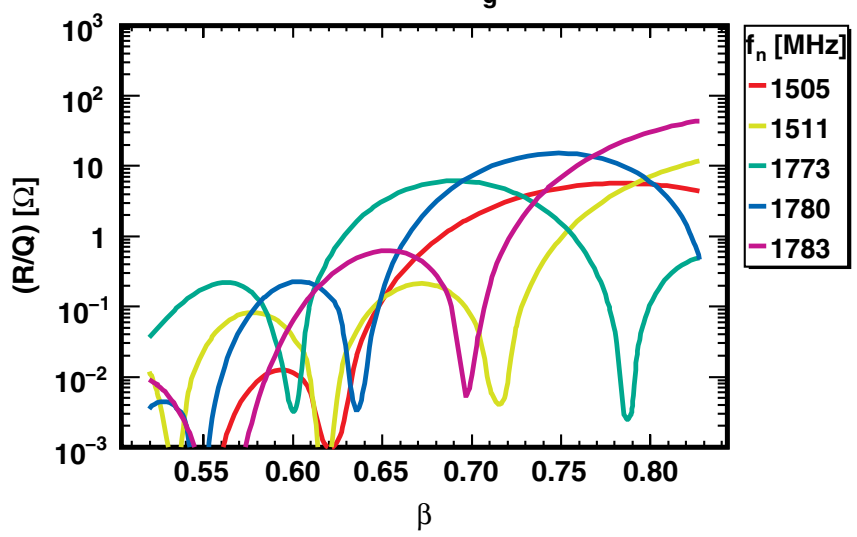

Dipole modes $\beta_{g}=0.65$ cavity

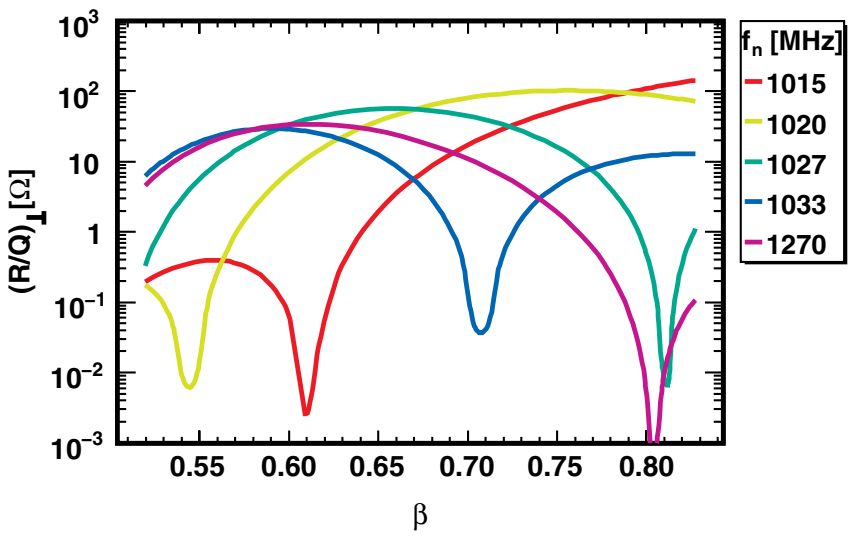

Monopole modes $\beta_{\mathrm{g}}=1$ cavity

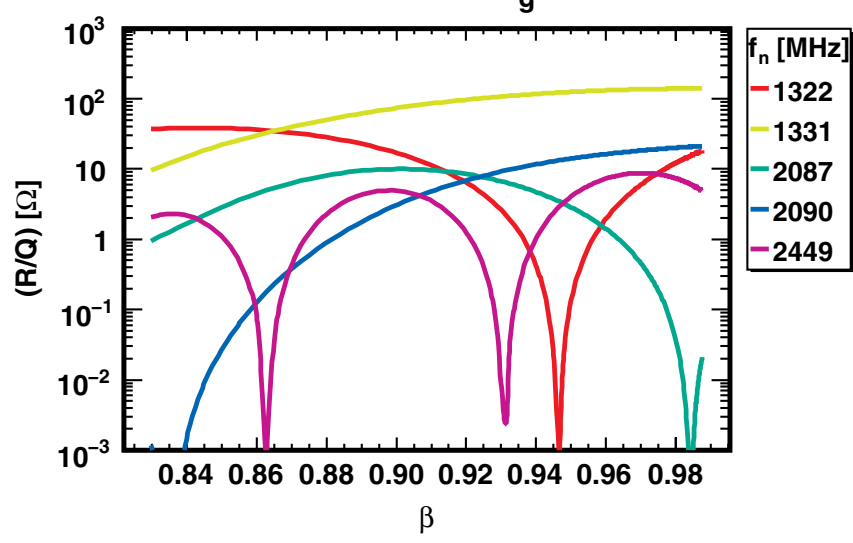

Dipole modes $\beta_{\mathrm{g}}=1$ cavity

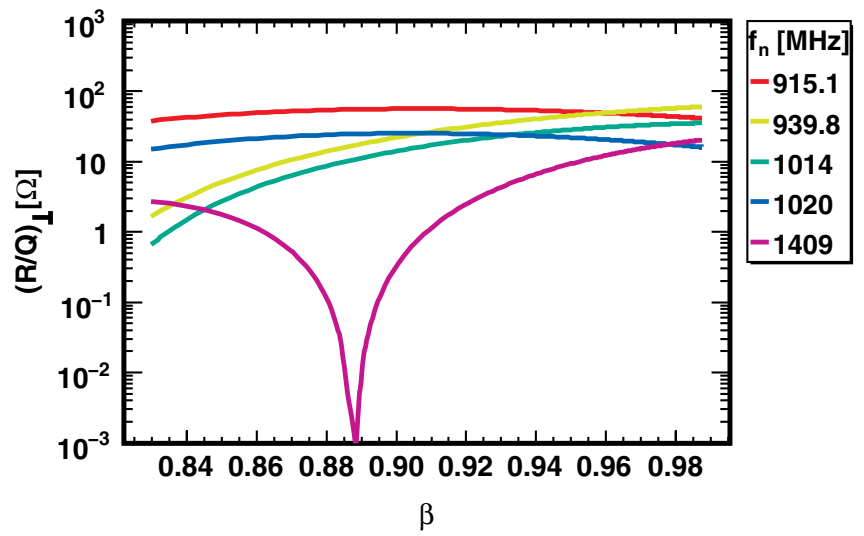

FIG. 3. $(R / Q)_{n}(\beta)$ maps of the monopoles and dipoles with high $(R / Q)_{n}$ values in the analyzed symmetric SPL cavities.

considered as independent and Gaussian distributed. Focusing in the longitudinal plane is achieved by the rf, which operates at $\phi=-15^{\circ}$ and the maximum zero current phase advance per period is limited to $75^{\circ}$. In order not to exceed this limit, the gradient in some cavities has to be reduced accordingly. A smooth focusing based on the phase advance per period for zero current in the transverse plane is used. Along the linac the phase advance per period decreases from $13.3^{\circ} / \mathrm{m}$ to $1.1^{\circ} / \mathrm{m}$ according to the used lattice design [48].

TABLE V. Beam input parameters and assumed variation based on Linac4 beam dynamics simulations and SNS data for the bunch charge scatter.

\begin{tabular}{lccc}
\hline \hline Parameter & & Value & $\sigma$ \\
\hline$E_{\text {input }}$ & {$[\mathrm{MeV}]$} & 160 & 0.078 \\
$\phi$ & {$[\mathrm{deg}$ at $704 \mathrm{MHz}]$} & -15 & 0.4 \\
$x$ & {$[\mathrm{~mm}]$} & 0.0 & 0.3 \\
$x^{\prime}$ & {$[\mathrm{mrad}]$} & 0.0 & 0.3 \\
$I_{b}$ & {$[\mathrm{~mA}]$} & 40 & $3 \%$ \\
\hline \hline
\end{tabular}

\section{Simulation execution}

The beam and HOM configuration, introduced before, are used to perform bunch tracking simulations in the longitudinal and transverse plane. Always at least two consecutive pulses are simulated. If the HOM voltage decay time $T_{d}$ gets longer than the period length ( $T_{r}=20 \mathrm{~ms}$ ) due to a high $Q_{\mathrm{ex}}$, the number of simulated pulses is increased until a steady state situation is reached, assuming an ideal loading curve from pulse to pulse. This is the case for $Q_{\mathrm{ex}}>10^{7}$. The number of simulated pulses is calculated using (7) and a theoretical load level of $99 \%$ :

$$
n_{\text {pulse }}>-\frac{T_{d}}{T_{r}} \ln (0.01)+1 .
$$

All results shown refer always to the last simulated pulse, if not stated differently.

\section{LONGITUDINAL PLANE}

Before looking at the effects of HOMs the nominal beam according to Table $\mathrm{V}$, with injection energy and phase jitter, is tracked through the linac without HOM interaction. The resulting energy and phase error distribution is plotted in a 


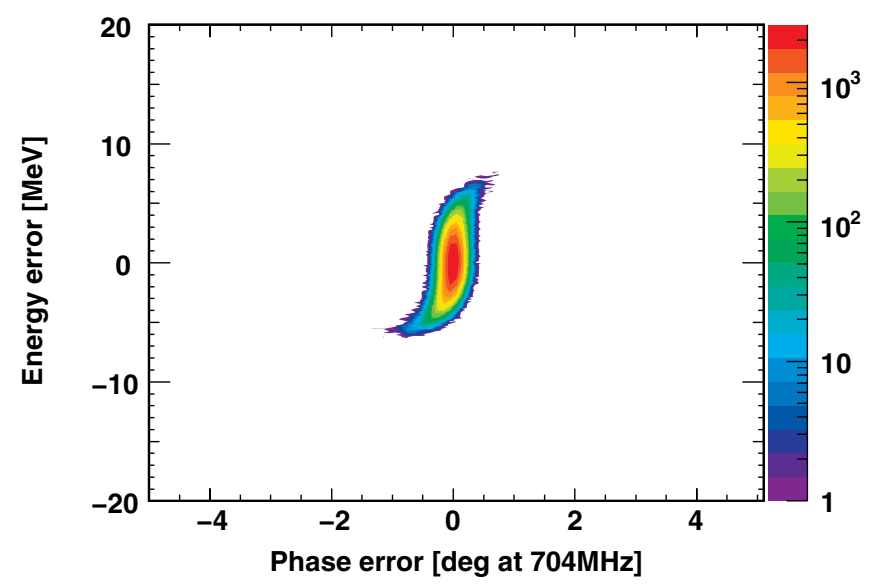

FIG. 4. 2D phase space histogram of one pulse at the end of the linac, which is not disturbed by HOMs. This distribution will be used as a reference later on. Settings: no $\mathrm{HOM}, N=1$, see Table VI.

2D phase space histogram, see Fig. 4. It shows no filamentation and a relatively sharp border contour line.

A set of default simulation values, listed in Table VI, is defined based on the data introduced before and will be used in all longitudinal beam dynamics simulations, if nothing different is stated.

\section{A. Initial simulation with a HOM}

The same bunch pattern as before is used, but an additional HOM monopole far away from any machine line is

TABLE VI. Default settings used in all longitudinal beam dynamics simulations.

\begin{tabular}{lccc}
\hline \hline Injected beam & & Mean & $\sigma$ \\
\hline$E_{\text {input }}$ & {$[\mathrm{MeV}]$} & 160 & 0.078 \\
$\phi$ & {$[\mathrm{deg}$ at $704 \mathrm{MHz}]$} & -15 & 0.4 \\
$x$ & {$[\mathrm{~mm}]$} & 0.0 & 0.0 \\
$x^{\prime}$ & {$[\mathrm{mrad}]$} & 0.0 & 0.0 \\
$I_{b}$ & {$[\mathrm{~mA}]$} & $400^{\mathrm{a}}$ & $3 \%$ \\
\hline Pulse structure & & & \\
\hline$T_{b}$ & {$[\mathrm{ps}]$} & 2.84 & \\
$T_{p}$ & {$[\mathrm{~ms}]$} & 1.00 & \\
$T_{r}$ & {$[\mathrm{~ms}]$} & 20.0 & \\
\hline Monopole mode $\beta_{g}=0.65 \mathrm{cavity}$ & & \\
\hline$f_{n}$ & {$[\mathrm{MHz}]$} & 1783 & 1 \\
$(R / Q)_{n}(\beta)$ & {$[\Omega]$} & $6 \times 10^{-5}-44$ & \\
$Q_{\mathrm{ex}}$ & $10^{7}$ & \\
\hline Monopole mode $\beta_{g}=1.0 \mathrm{cavity}$ & & \\
\hline$f_{n}$ & {$[\mathrm{MHz}]$} & 1331 & 1 \\
$(R / Q)_{n}(\beta)$ & {$[\Omega]$} & $10-140$ & \\
$Q_{\mathrm{ex}}$ & & $10^{7}$ & \\
\hline \hline
\end{tabular}

${ }^{\mathrm{a}}$ Ten times nominal current as safety margin. present in each cavity, see Table VI. The case where a HOM falls on a machine line will be discussed later in Sec. V G. The beam current is $\left\langle I_{b}\right\rangle=400 \mathrm{~mA}$, the charge scatter $\sigma_{q}=3 \%$, the $\mathrm{HOM}$ frequency spread $\sigma_{f_{\text {ном }}}=$ $1 \mathrm{MHz}$, and $Q_{\mathrm{ex}}=10^{7}$. The simulation is repeated for 1000 linacs, which means that each time a different seed for the HOM frequency spread generator is used. All other parameters stay the same. In Fig. 5 the resulting phase space histogram is shown. The mean values in both planes show only minor deviations in the order of $10^{-3}$ compared to without HOM, and slight changes are visible in the border area.

The average and maximum HOM voltage, present in the cavities after the second pulse, is illustrated in Fig. 6 . The voltage changes along the linac according to

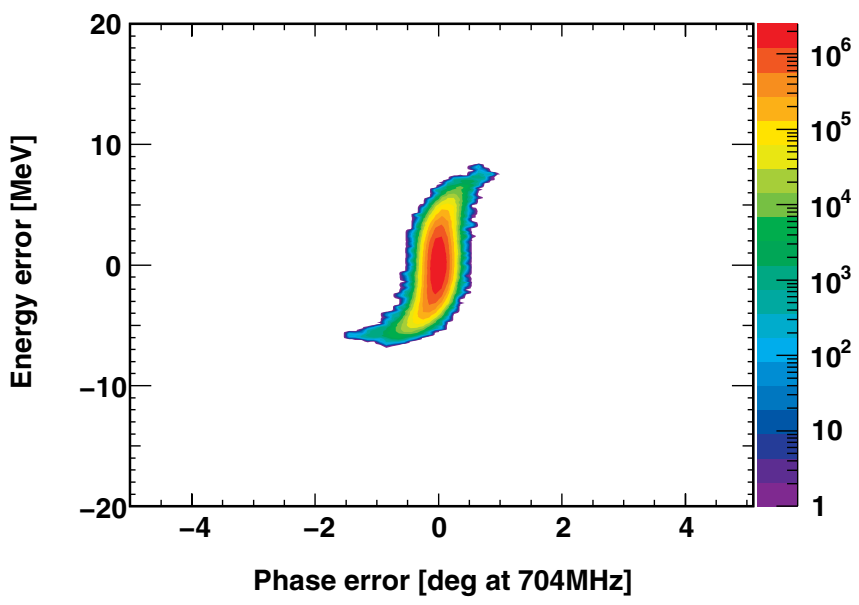

FIG. 5. 2D phase space histogram at the exit of 1000 linacs, where always the same pulse was tracked through, but the seed for the HOM frequency distribution was varied. Settings: $N=1000$, see Table VI.

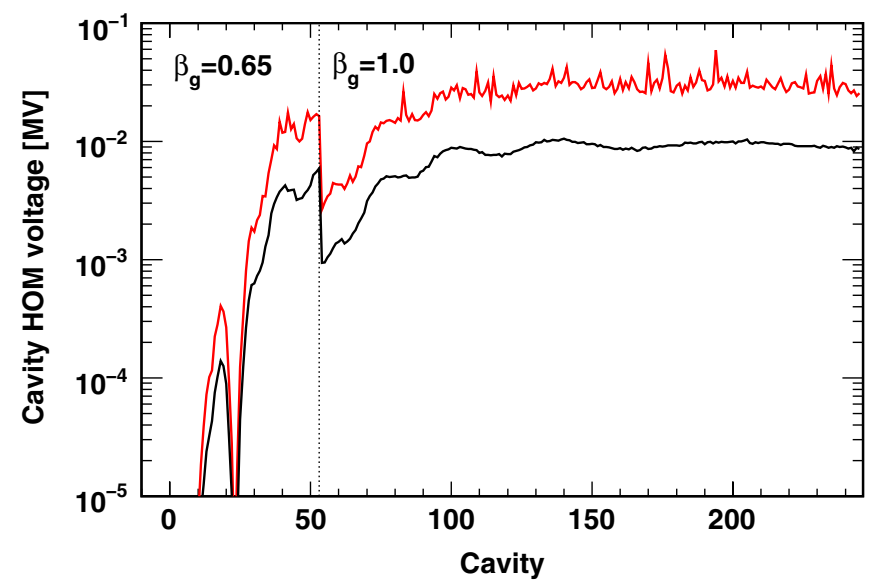

FIG. 6. Average (black) and maximum (red) HOM voltage present in the cavities after two pulses. The $(R / Q)_{n}(\beta)$ dependency is clearly visible in the medium beta section. Settings: $N=1000$, see Table VI. 
the $(R / Q)_{n}(\beta)$ change. The maximum values are about 5 times higher than the average values.

In order to compare the results obtained with different input parameters and to identify easily the impact of the HOM, a figure of merit has to be defined. The phase space area created by all bunches in a pulse at the end of the linac

$$
\epsilon=\pi \sqrt{\left\langle d E^{2}\right\rangle\left\langle d \phi^{2}\right\rangle-\langle d E d \phi\rangle^{2}},
$$

where $d E$ is the energy error of a bunch and $d \phi$ its phase error, is used for this purpose. This value is rotation but not displacement invariant, which means the energy and phase oscillations along the linac have no effect on $\epsilon$ but a general energy or phase offset increases it.

A growth $\epsilon_{\mathrm{HOM}} / \epsilon$ with a growth rate $\epsilon_{\mathrm{HOM}} / \epsilon-1$ is then defined as the effective ratio of a HOM disturbed pulse over an undisturbed pulse. This can be used to measure the influence of HOMs or rf errors.

\section{B. rf errors without HOMs}

One of the main sources of energy and phase jitter are the rf power sources as already reported [49]. The SPL design aims for RMS errors of $0.5^{\circ}$ in phase and $0.5 \%$ in amplitude. With these errors and assuming a uniform distribution 1000 different linacs are simulated. The phase space distribution at the exit of the linac is shown in Fig. 7. A significant phase space area increase can be observed as compared to the case where no rf errors are present. The distribution of $\epsilon$ is normalized to the case, where no $\mathrm{rf}$ errors are present. On average the phase space increases about a factor 3.8, which will be used as the tolerable limit for HOM induced beam degradation.

The values of the energy and phase error at the end of the linac are about a factor 2 larger as compared to the values in [2]. This difference can be explained with a different

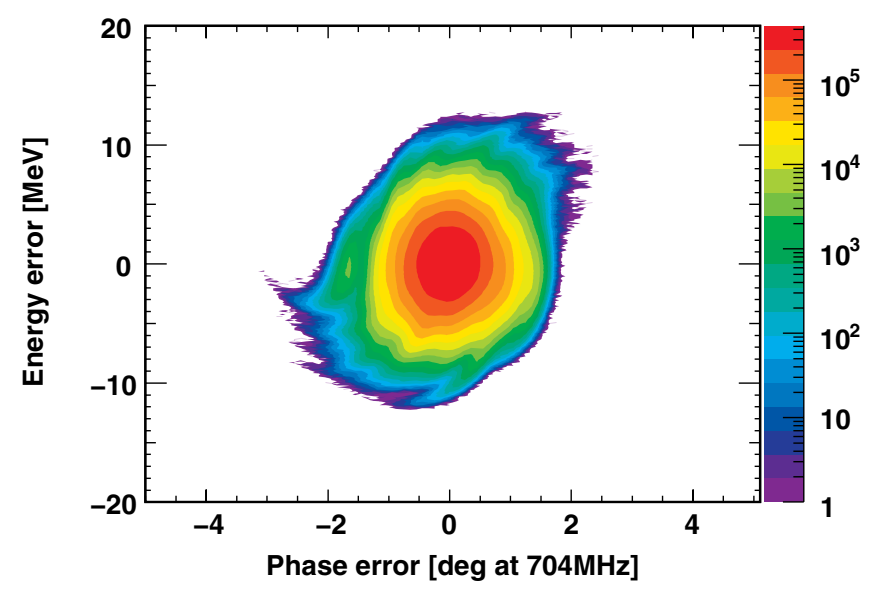

FIG. 7. 2D phase space histogram at the exit of 1000 linacs with rf errors, but no HOM present. The phase space area is increased significantly compared to Fig. 5. Settings: uniform distributed $\mathrm{rf}$ errors $\left(0.5^{\circ}\right.$ in phase and $0.5 \%$ in magnitude), no HOM, $N=1000$, see Table VI. energy gain per cavity due to energy error caused transit time factor changes. This effect was not included in the previous study. To confirm this explanation, simulations, using the energy gain of the synchronous particle per cavity for each particle, are executed and lead to the expected energy and phase error as stated in [2]. Further simulations with different rf errors and the influence of HOMs will be shown later in Sec. V I.

\section{Current and damping sweeps}

The first two parameters which are investigated in detail are the beam current $\left\langle I_{b}\right\rangle$ and the external damping $Q_{\mathrm{ex}}$. Figure 8 shows a parameter sweep, where $\left\langle I_{b}\right\rangle$ is varied between 40 and $400 \mathrm{~mA}$ and $Q_{\mathrm{ex}}$ between $10^{4}$ and $10^{8}$. Each set is simulated for 100 linacs. At nominal current, no obvious change is visible. At $Q_{\mathrm{ex}}=10^{7}, \epsilon_{\mathrm{HOM}} / \epsilon-1$ is about $0.2 \%$. Increasing the beam current by a factor of 10 leads to an increase of $\epsilon_{\mathrm{HOM}} / \epsilon-1$ by a factor 100, but only $\sim 3 \%$ in absolute terms. Below $Q_{\mathrm{ex}}=10^{5}$ even at $400 \mathrm{~mA} \epsilon_{\mathrm{HOM}} / \epsilon-1$ is less than $1 \%$.

In general, $\epsilon_{\mathrm{HOM}} / \epsilon$ is proportional to $\left\langle I_{b}\right\rangle^{2}$, while the HOM voltage increase is proportional to $\left\langle I_{b}\right\rangle$, because the HOM voltage induced by a single bunch is proportional to the charge per bunch, see Eq. (1). This is no contradiction, because $\epsilon$ is the correlated product of the energy and phase error and the mean value of both quantities increases linear with $\left\langle I_{b}\right\rangle$.

Looking now at the $Q_{\text {ex }}$ dependency, one can divide the graph into three regions: $Q_{\mathrm{ex}}<4 \times 10^{6}, 4 \times 10^{6}<Q_{\mathrm{ex}}<$ $6 \times 10^{7}$, and $6 \times 10^{7}<Q_{\mathrm{ex}}$. In the second region around $Q_{\text {ex }} \approx 4 \times 10^{7}$, a plateau is visible. This can be explained by the fact that the rise time to reach the maximum HOM voltage is longer than the beam pulse, but the decay time is shorter than the period length. So the voltage decays

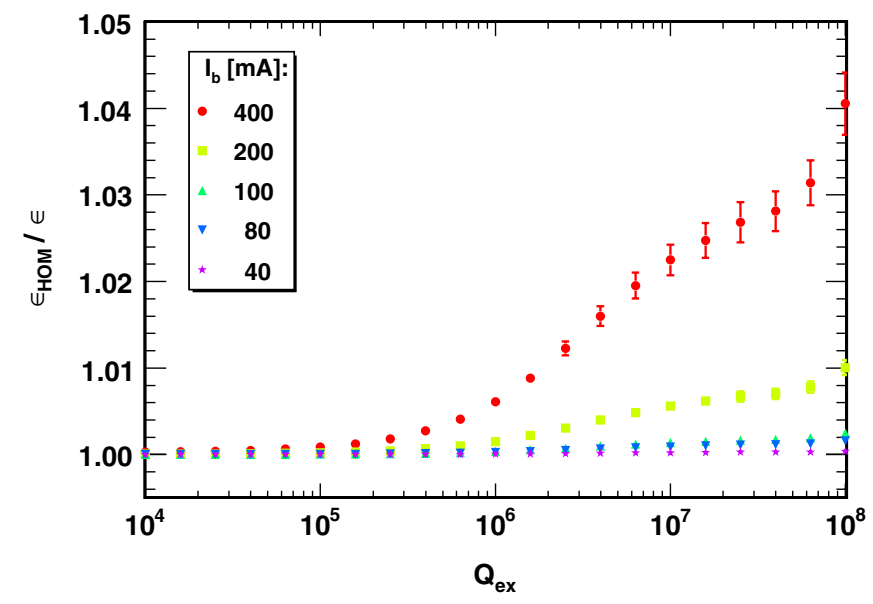

FIG. 8. Average longitudinal phase space increase and deviation of 100 linacs against the damping $Q_{\mathrm{ex}}$ for different $\left\langle I_{b}\right\rangle$. $\epsilon_{\mathrm{HOM}} / \epsilon$ is proportional to $\left\langle I_{b}\right\rangle^{2}$. The plateau around $Q_{\mathrm{ex}} \approx 4 \times$ $10^{7}$ is due to the pulse period structure. Settings: $N=100$, see Table VI. 
completely before the next pulse arrives. In the first region, the maximum HOM voltage is reached during the pulse. If $Q_{\text {ex }}$ increases further (third region), the decay time gets longer than a period length and the HOM voltage can increase from pulse to pulse. This damping regime should be avoided to prevent long term instabilities. This characteristic curve of the $Q_{\mathrm{ex}}$ dependency shows the same qualitative behavior as the square of the voltage calculated with Eq. (8), taking only the real part and neglecting the oscillations introduced by the imaginary part.

In all further simulations, 10 times the nominal beam current $\left\langle I_{b}\right\rangle=400 \mathrm{~mA}$ is used as a safety margin and a $Q_{\text {ex }}=10^{7}$ is assumed.

\section{Beam injection noise}

The excitation of a HOM far from a resonance depends strongly on the beam noise. In order to verify that the used injection beam noise pattern is a representative sample, 1000 simulations are carried out, where all the machine parameters stay the same, but the injection beam noise pattern is varied. Then the simulations are repeated, where additionally the HOM frequency pattern is varied. There is only a minor difference in these simulation results compared to the result, where a fixed injection beam noise pattern is used and the HOM frequency pattern is varied. Hence, the same injection beam noise pattern will be used in all further simulations.

\section{E. Charge scatter}

The number of particles per bunch is not constant over time and so there is a bunch to bunch charge scatter, which is assumed to be Gaussian distributed with a sigma of a few percent. This scatter has an impact on the HOM excitation and induces a beam blowup as shown in Fig. 9, where $\sigma_{q}$ is varied between 0 and $10 \%$. For each charge scatter sigma

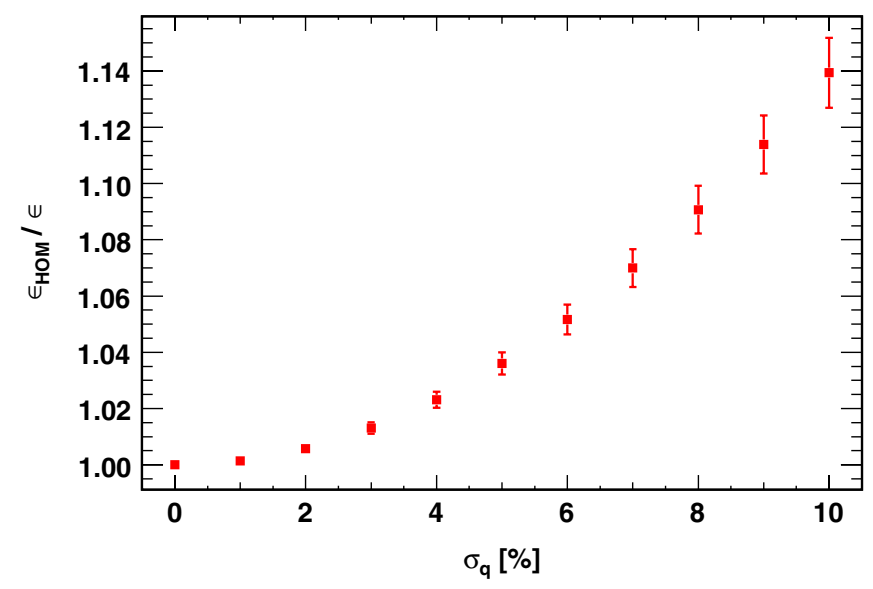

FIG. 9. Average longitudinal phase space increase and deviation as a function of the Gaussian bunch to bunch charge scatter. The phase space increases approximately with $\sigma_{q}^{2}$. Settings: $N=100$, see Table VI.
100 different linacs with a $\sigma_{f_{\text {ном }}}=1 \mathrm{MHz}$ are simulated. A change in the used charge scatter pattern has only a minor influence and can be neglected in the further discussion. The observed increase in $\epsilon_{\mathrm{HOM}} / \epsilon$ is proportional to $\sigma_{q}^{2}$ and scales with the beam current in the same way.

\section{F. HOM frequency spread}

Past studies $[7,8]$ have shown that the HOM frequency spread plays an important role in building up beam instabilities. In the simulation presented here, the parameters listed in Table VI are used, but the width of the Gaussian distributed HOM frequency spread $\sigma_{f_{\text {Ном }}}$ is varied in the range of $10^{4}-10^{7} \mathrm{~Hz}$ around the nominal HOM frequencies. In total 100 linacs are simulated in each step.

The average and maximum value of $\epsilon_{\mathrm{HOM}} / \epsilon$ is plotted in Fig. 10, where a significant growth is observed for $\sigma_{f_{\text {ном }}}<$ $100 \mathrm{kHz}$. A larger HOM frequency spread leads to less beam perturbation and reaches a minimum level for $\sigma_{f_{\text {ном }}}>1 \mathrm{MHz}$. In this regime, there is no constructive interaction of the HOMs in the single cavities.

At nominal beam current, the impact is less than $1 \%$ at $\sigma_{f_{\text {ном }}}=10 \mathrm{kHz}$. The expected HOM frequency spread in the SPL cavities is assumed to be greater than $1 \mathrm{MHz}$ for all modes beside the fundamental passband and is well above the threshold.

No result for $\sigma_{f_{\text {ном }}}=10 \mathrm{MHz}$ is shown in Fig. 10, because the HOM frequency in the medium beta section would be less than $3 \sigma_{f_{\text {ном }}}$ away from the 5 th machine line and would disturb the result. The effect of HOMs at a machine line is discussed in the next subsection.

The simulations are also carried out for stronger damping (lower $Q_{\text {ex }}$ ). This leads to a shift of the HOM frequency spread threshold to lower $\sigma_{f_{\text {ном }}}$. For higher $Q_{\text {ex }}$ the threshold moves to larger $\sigma_{f_{\text {ном }}}$. A comparison with analytic results is done in Sec. VIE.

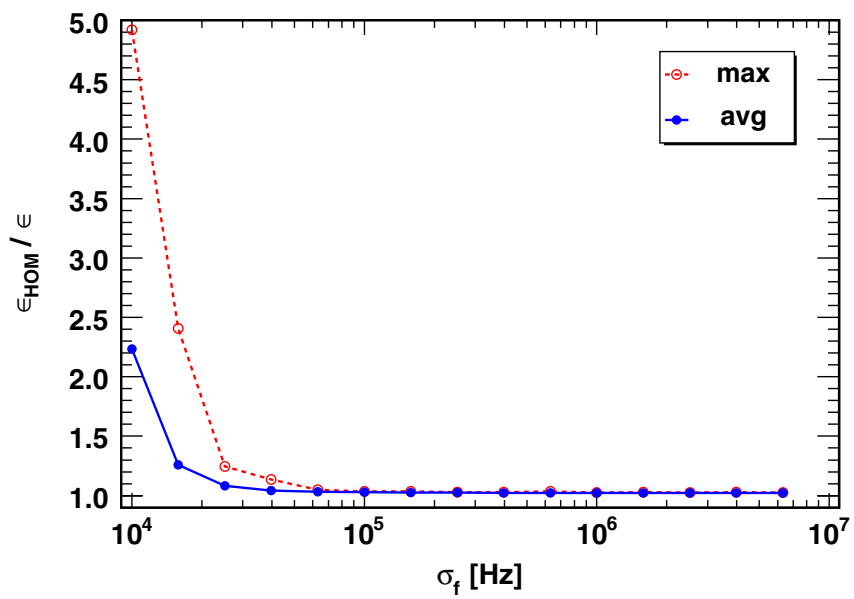

FIG. 10. Average (solid, blue) and maximum (dashed, red) longitudinal phase space increase against the HOM frequency spread for 100 linacs. Settings: $N=100$, see Table VI. 


\section{G. Machine lines}

The most dangerous situation is a HOM which falls on a machine line (ML). In this case a resonance excitation from bunch to bunch occurs and a significant HOM voltage can build up in the cavities. The minimum distance of a $\mathrm{HOM}$ to a machine line is estimated, where operation with no essential impact by HOMs is possible. Therefore, two sets of simulations are carried out. First, the mean HOM frequency $\left\langle f_{\mathrm{HOM}}\right\rangle$ is set on a machine line and the HOM frequency spread $\sigma_{f_{\text {ном }}}$ is increased. Second, $\left\langle f_{\text {HOM }}\right\rangle$ is shifted away from the machine line with a constant $\sigma_{f_{\text {ном }}}$. Additionally the effect of strong HOM damping is analyzed. In both linac sections the $(R / Q)_{n}(\beta)$ maps of the monopole modes listed in Table $\mathrm{V}$ are used as before, but the HOM frequency is shifted to a machine line. The beam current is reduced to the nominal $40 \mathrm{~mA}$, because the beam is lost immediately at $400 \mathrm{~mA}$ in almost all cases. All other simulation parameters are the default ones listed in Table VI

\section{HOM frequency spread at a machine line}

The mean HOM frequency is set to the 4th machine line at $1408.8 \mathrm{MHz}$ and the HOM frequency spread is varied from $10 \mathrm{kHz}$ to $10 \mathrm{MHz}$. In order to have enough statistics, 100 linacs are simulated in each step and the resulting $\epsilon_{\mathrm{HOM}} / \epsilon$ against $\sigma_{f_{\text {ном }}}$ is shown in Fig. 11, where the beam is strongly disturbed and beam losses occur for $\sigma_{f_{\text {ном }}}<400 \mathrm{kHz}$. Even for a large HOM frequency spread of $10 \mathrm{MHz}$ there is still a significant impact. One cavity with a HOM at the machine line can drive instabilities as already shown earlier in this paper. Hence, a HOM with a high $(R / Q)_{n}(\beta)$, sitting directly on a machine line, has to be excluded during the cavity design phase.

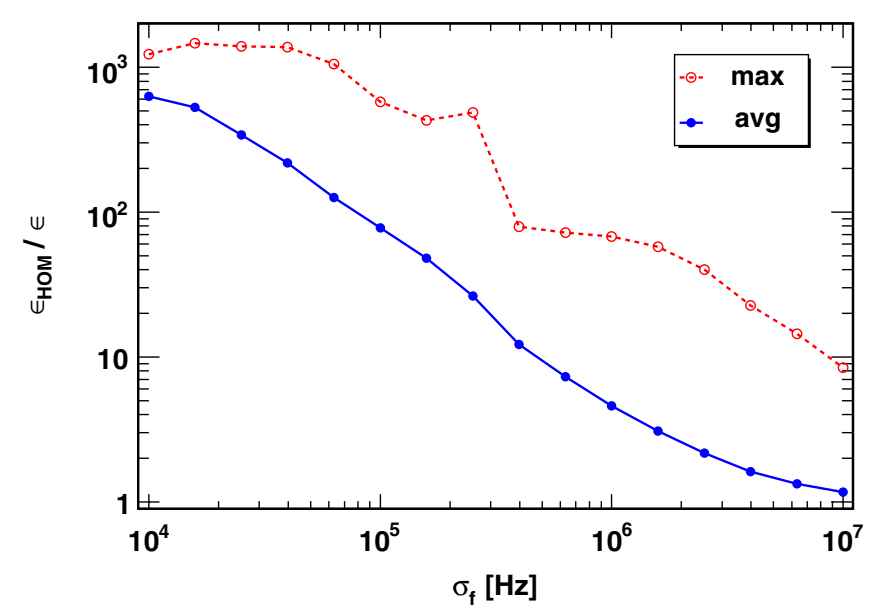

FIG. 11. Average (solid, blue) and maximum (dashed, red) longitudinal phase space increase as a function of the HOM frequency spread at the 4th machine line. Losses occur in the case of $\epsilon_{\mathrm{HOM}} / \epsilon>100$. Settings: $\left\langle f_{\mathrm{HOM}}\right\rangle=1408.8 \mathrm{MHz},\left\langle I_{b}\right\rangle=$ $40 \mathrm{~mA}, N=100$, see Table VI.

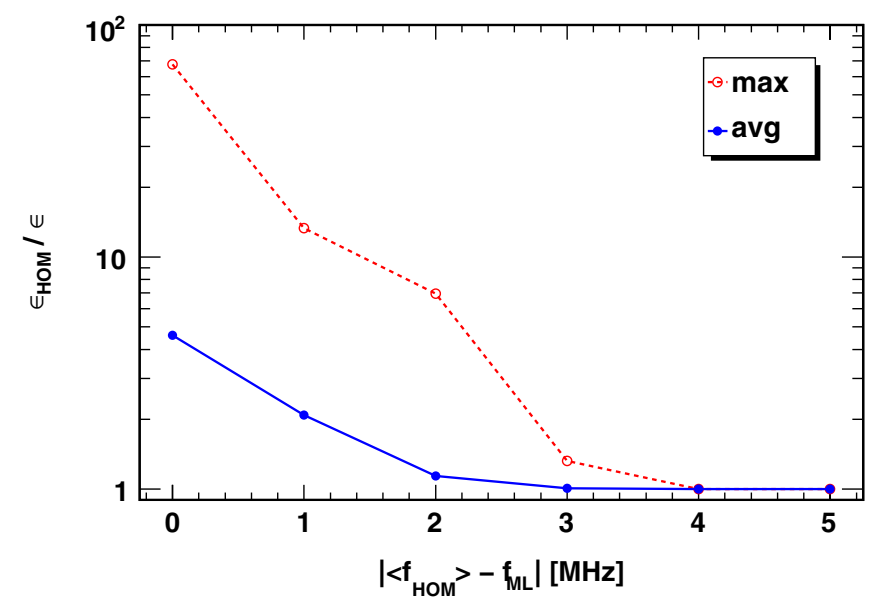

FIG. 12. Average (solid, blue) and maximum (dashed, red) longitudinal phase space increase as a function of the distance to 4th machine line. Settings: $\left\langle f_{\mathrm{HOM}}\right\rangle$ shifted, $\left\langle I_{b}\right\rangle=40 \mathrm{~mA}$, $N=100$, see Table VI.

\section{HOM frequency distance to a machine line}

The HOM frequency spread is fixed to $1 \mathrm{MHz}$ and $\left\langle f_{\mathrm{HOM}}\right\rangle$ increased in $1 \mathrm{MHz}$ steps starting at the 4th machine line. The results are shown in Fig. 12. Outside the $3 \sigma$ area which corresponds to $3 \mathrm{MHz}$, there is no impact of the machine line any more and the normal off resonance growth is observed. Since the machine line resonances are very sharp, only very few cavities with a HOM in this small bandwidth are needed to drive beam instabilities. If the distance of the mean HOM frequency together with its spread is larger than the bandwidth of the resonance, no resonance excitation can occur. This is the case here for $\left|\left\langle f_{\mathrm{HOM}}\right\rangle-f_{\mathrm{ML}}\right|>3 \mathrm{MHz}$. This criteria can be used in the cavity design phase to exclude frequency bands around the machine lines.

\section{Strong damping}

Also the dependency on $Q_{\mathrm{ex}}$ in case of a resonance excitation is investigated. The average and maximum phase space increase of 100 linacs is shown in Fig. 13, where the damping and the current is varied. At $400 \mathrm{~mA}$ the phase space increase is always higher than the increase due to rf errors above $Q_{\mathrm{ex}}=10^{4}$. Decreasing the current to the nominal $40 \mathrm{~mA}$ relaxes the situation, but even there the maximum phase space increase exceeds the average phase space increase due to rf errors above $Q_{\mathrm{ex}}=10^{5}$. Even with strong HOM damping, operation is critical from the beam dynamics point of view, if a HOM falls on a principal machine line. In this case also the power dissipated in the HOM coupler is significant and has to be considered.

\section{Resonance in a single cavity}

In the subsections before the mean HOM frequency was set to a machine line. Now only one cavity in the linac has a 


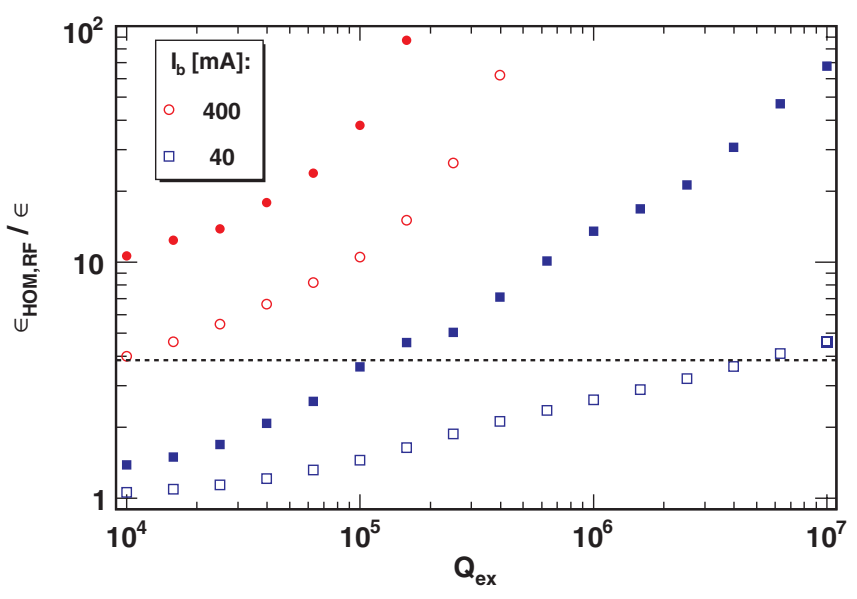

FIG. 13. Average (hollow dots) and maximum (solid dots) longitudinal phase space increase as a function of the damping, where a HOM is resonantly excited. The average increase due to rf errors is indicated with the dashed line. Settings: $\left\langle f_{\text {HOM }}\right\rangle=1408.8 \mathrm{MHz}, N=100$, see Table VI.

HOM directly at the 8th machine line with a $(R / Q)_{n}=$ $1 \Omega$. In all other cavities the nominal HOM with a frequency spread of $1 \mathrm{MHz}$ is present. A sweep is performed where the resonant HOM is put in each cavity in the linac with a $Q_{\mathrm{ex}}=10^{8}$. Four consecutive pulses are simulated to take into account the long rise time at that damping level. For comparison also the voltages and the phase space increase after the first pulse is recorded. The HOM voltage present in the resonantly excited cavity is about $336 \mathrm{kV}$ after the first pulse and $405 \mathrm{kV}$ after the last pulse, which is in good agreement with the analytical value obtained by Eq. (8). There is only a minor deviation $\left(\sim 10^{-3}\right)$ in the resonance HOM voltage along the linac due to the arrival time error.

In Fig. 14 the resulting phase space increase is shown as a function of the cavity, where the HOM at the machine line is located. The strongest influence is observed as expected, if the resonant HOM is at the beginning of the linac, because the relative deceleration kick [50], caused by the HOM, is highest there compared to the particle energy. Oscillations in phase space growth along the linac are observed, which can be explained by the following. Bunches with an energy and phase error perform oscillations in the phase space traveling along the linac due to the rf focusing. The used injection pattern causes a characteristic oscillation with peaks in the phase and energy error at certain points along the linac. If now the resonant HOM is at a position with a low energy error, the impact is higher than at positions with high energy error. The period length of the observed phase space increase oscillations fits with the phase error oscillation. In a real machine these peaks in energy and phase error are not fixed due to a changing injection distribution. Hence, the envelope of the oscillation should be taken as a measure of the phase space increase. The influence at the first pulse is less, because

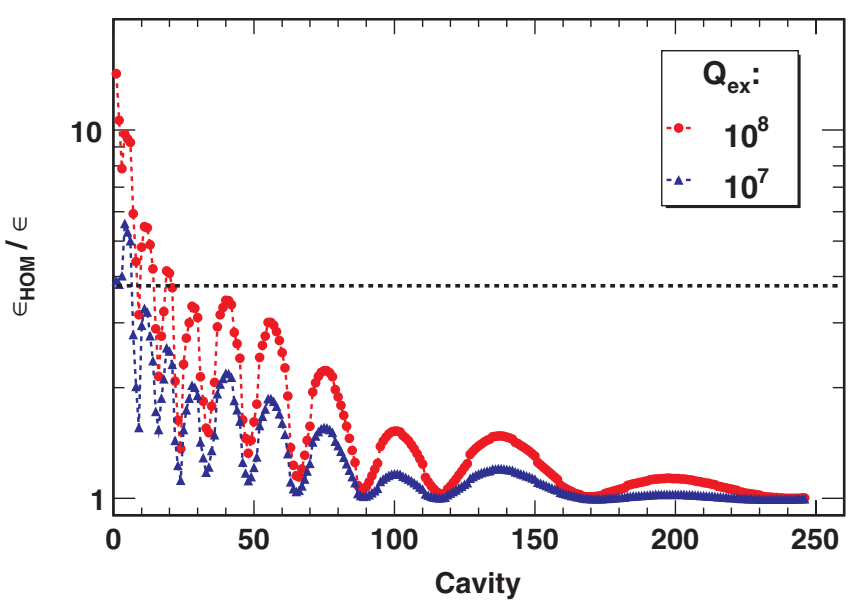

FIG. 14. Longitudinal phase space increase as a function of the cavity, where the HOM at the 8th machine line is located for different damping. The average increase due to $\mathrm{rf}$ errors is indicated with the dashed line. An oscillating phase space increase is observed, which decreases further downstream in the linac. The oscillation is due to the used injection noise pattern. Settings: $f_{\mathrm{ML}}=2817.6 \mathrm{MHz},(R / Q)_{\mathrm{ML}}=1 \Omega, N=1$, other cavities use default HOM configuration, see Table VI.

at the start no HOM voltage is present in the cavity. At $Q_{\text {ex }}=10^{8}$ the decay time is larger than the repetition period and the HOM voltage cannot decay completely between two pulses. Hence, the HOM voltage increases from pulse to pulse and causes a larger phase space increase.

The total phase space increase due to a resonant HOM with a low $(R / Q)_{n}(<1)$, can be tolerated at a damping of $Q_{\text {ex }}=10^{8}$ in the high beta section, without any major influence on the beam. In the beginning of the medium beta section the phase space increase is significantly larger than the increase due to rf errors. This situation improves, if a higher damping is present or the mode is detuned. Detuning the resonant mode leads also to a decrease of the maximum HOM voltage and causes less beam disturbance. The HOM voltage is reduced about a factor 10 , if the mode frequency is shifted by $1 \mathrm{kHz}$ at $Q_{\mathrm{ex}}=10^{8}$. A cavity in operation is tuned each pulse to compensate for the Lorentz force detuning. It is very unlikely that a HOM stays over several pulses exactly on a machine line. Hence, the most critical case is the HOM voltage built up during one pulse. The HOM voltage induced during one pulse saturates above $Q_{\text {ex }}=10^{8}$ due to the long filling time. From this point of view, a trapped mode with a $(R / Q)_{n}<1$ is only a concern for the beam stability, if it appears in the beginning of the medium beta section and its frequency meets exactly a machine line. The width of the resonance decreases with increasing $Q_{\mathrm{ex}}$. Hence, the needed detuning decreases as well. The exact limits for $(R / Q)_{n}(\beta)$ as a function of the resonance frequency can be calculated analytically using Eq. (8) and limiting the HOM voltage to a value smaller than the accelerating voltage error caused by the rf system. 
TABLE VII. Chopping patterns used in the beam dynamics simulations.

\begin{tabular}{lc}
\hline \hline Chopping pattern $(m / N)$ & $f_{c}[\mathrm{MHz}]$ \\
\hline $5 / 8$ & 44.025 \\
$50 / 80$ & 4.4025 \\
$500 / 800$ & 0.44025 \\
\hline \hline
\end{tabular}

\section{H. Substructured pulses}

Any substructure in the beam pulse, created by chopping, introduces new spectral lines in addition to the machine lines created by the bunch spacing $\left(n f_{b}\right.$, $n \in \mathbb{N}$ ). The chopping frequency $f_{c}=f_{b} / N$ is defined by the periodicity of the substructure, where $m$ out of $N$ bunches are used. To keep all possibilities open and to allow any chopping pattern means that stable operation with a resonant HOM excitation must be guaranteed. Therefore, three different $(m / N)$ patterns with the same ratio and a bunch repetition frequency of $352.2 \mathrm{MHz}$ are investigated in more detail and listed in Table VII. The charge per bunch is increased by a factor $8 / 5$ to keep the total charge per pulse constant.

A frequency scan between the 3rd and 4th machine line is performed where the mean HOM frequency with a $\sigma_{f, \mathrm{HOM}}=1 \mathrm{MHz}$ is set to a chopping machine line and the $(R / Q)_{n}(\beta)$ maps of the monopole modes listed in Table V are used.

The longitudinal phase space increase of one simulated pulse at nominal beam current and $Q_{\mathrm{ex}}=10^{7}$ versus the frequency is shown in Fig. 15, where also the simulated $\mathrm{TM}_{011}$ monopole frequencies of the $\beta_{g}=1$ cavity are

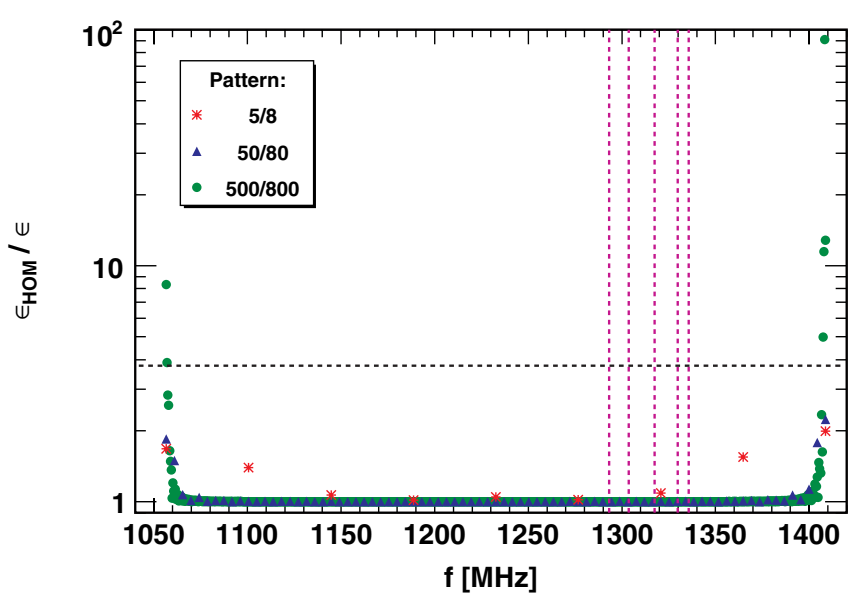

FIG. 15. Longitudinal phase space increase against the HOM frequency, where a HOM falls on a chopping machine line at nominal current and $Q_{\mathrm{ex}}=10^{7}$ for different chopping patterns. The rf induced growth (black dashed line) is indicated and the found monopole modes (violet dashed lines) in the investigated frequency range. Settings: chopping, $\left\langle f_{\mathrm{HOM}}\right\rangle$ shifted, $\left\langle I_{b}\right\rangle=$ $40 \mathrm{~mA}, N=1$, see Table VI.

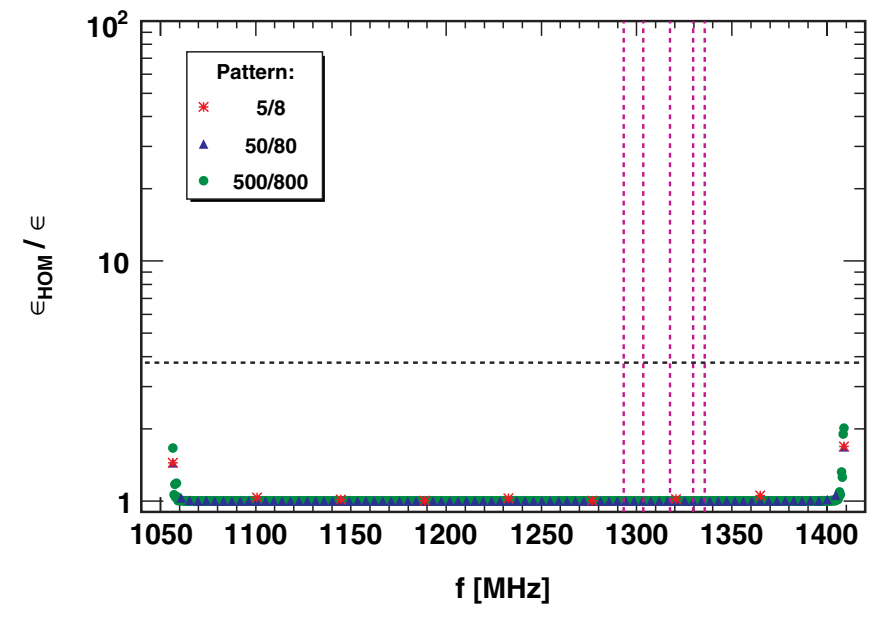

FIG. 16. Same as Fig. 15, but with strong HOM damping. Settings: chopping, $\left\langle f_{\mathrm{HOM}}\right\rangle$ shifted, $\left\langle I_{b}\right\rangle=40 \mathrm{~mA}, Q_{\mathrm{ex}}=10^{5}$, $N=1$, see Table VI.

indicated along with the phase space increase due to rf errors. Away from the principal machine lines only the $5 / 8$ pattern triggers a certain growth. One of the $5 / 8$ chopping resonance lines is close to the expected frequency of the $\mathrm{TM}_{011,3 / 5 \pi}$ mode, which has a maximum $(R / Q)_{n}$ value of about $5 \Omega$. In general, the impact of the chopping machine lines on beam degradation increases with the repetition rate, which can be explained by looking at its Fourier components. At $Q_{\mathrm{ex}}=10^{5}$ no growth due to the chopping machine lines is observed in Fig. 16 for all chopping patterns at nominal current, which ensures stable operation for any chopping pattern. At this damping level only principal machine lines can cause beam blowup.

The pulse substructure used at SNS [18] corresponds to a $260 / 378$ chopping pattern. There the repetition rate is too small to create any potentially dangerous chopping machine lines. Hence, only the principal machine lines can drive instabilities significantly. Since all monopole modes in the SNS cavities [51] are far away from these resonance lines, no resonant HOM excitation is observed during operation [30].

\section{I. rf errors and HOMs}

As already introduced, rf errors lead to a significant longitudinal phase space increase. Repeating the 1000 simulations with an equal distributed RMS rf error of $0.5^{\circ}$ in phase and $0.5 \%$ in amplitude, but additionally with a HOM present, leads to the phase space histogram shown in Fig. 17. No changes as compared to Fig. 7 are observed and the HOM does not drive any further beam blowup.

Increasing the rf error leads to a further increase in $\epsilon$, as shown in Fig. 18. Also in this case there is no deviation observed, if additionally a HOM is present. 


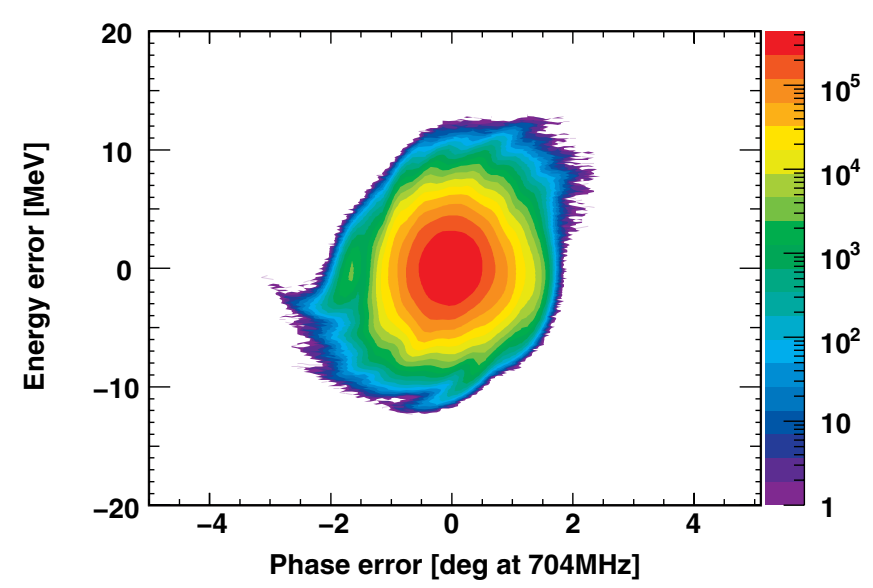

FIG. 17. 2D phase space histogram at the exit of 1000 linacs, where rf errors and one HOM is present. There is no significant change in the phase space histogram compared to Fig. 7. Settings: uniform distributed rf errors $\left(0.5^{\circ}\right.$ in phase and $0.5 \%$ in magnitude), $N=1000$, see Table VI.

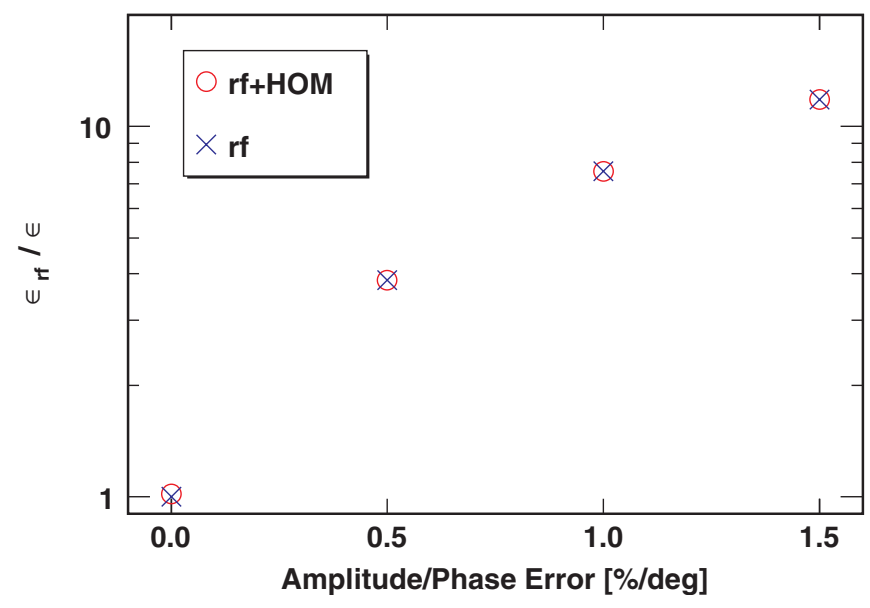

FIG. 18. Average longitudinal phase space increase as function of the rf error with and without a HOM. Settings: uniform distributed rf errors, $N=1000$, see Table VI.

The effect of a HOM at a machine line together with $\mathrm{rf}$ errors dependent on the damping is studied as well. To better illustrate the effect of a HOM at a machine line in combination with rf errors ( $\mathrm{rf}+\mathrm{ML}$ ), the following additional cases are simulated: only a HOM at a machine line (ML), only rf errors (rf), and rf errors with a HOM not resonantly excited ( $\mathrm{rf}+\mathrm{HOM})$ are plotted together in Fig. 19. There the average and maximum values of 100 simulations per damping value are shown, where the HOM frequency pattern and the rf error pattern are both varied. An equal distributed RMS rf error of $0.5^{\circ}$ in phase and $0.5 \%$ in amplitude is used in all simulations with rf errors.

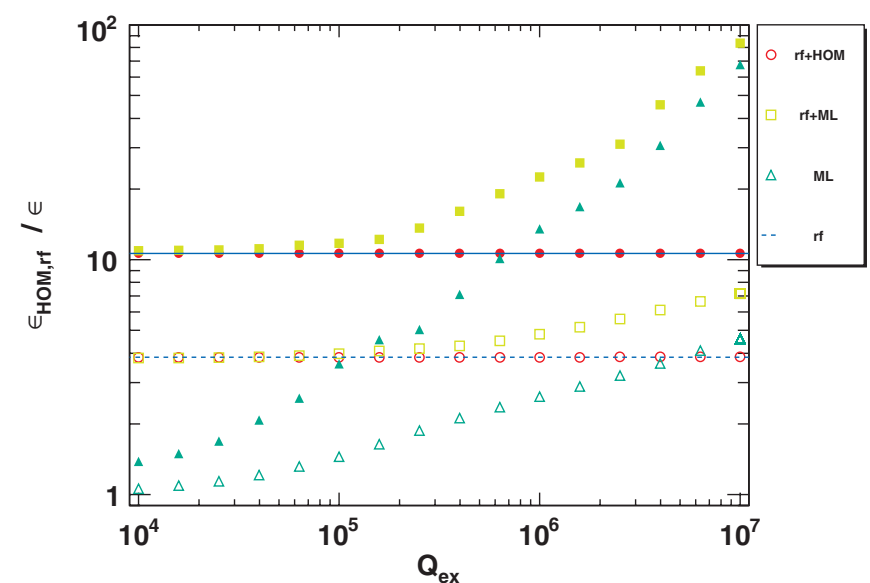

FIG. 19. Average (hollow dots) and maximum (filled dots) phase space increase against the damping $Q_{\mathrm{ex}}$ at nominal current for different cases, where rf errors (rf) and/or a HOM at a machine (ML) or off resonance (HOM) is present. The average (dashed line) and maximum (solid line) increase due to pure rf errors is also included. Settings: uniform distributed rf errors $\left(0.5^{\circ}\right.$ in phase and $0.5 \%$ in magnitude $),\left\langle I_{b}\right\rangle=40 \mathrm{~mA}$, $\left\langle f_{\mathrm{HOM}, \mathrm{ML}}\right\rangle=1,408.8 \mathrm{MHz}, N=100$, see Table VI.

At $Q_{\mathrm{ex}}=10^{4}$ the HOM at a machine line is damped strong enough not to cause an additional phase space increase. Between $10^{4}<Q_{\mathrm{ex}}<10^{5}$ the influence of the HOM starts to rise and the maximum values of $\epsilon$ increase slightly, while the average values are still the same as for the other cases. Above $Q_{\mathrm{ex}}=10^{5}$ the effective phase space starts to increase significantly and the HOM at the machine line becomes the main source of beam blowup, if the damping is less than $Q_{\mathrm{ex}}=5 \times 10^{6}$.

\section{J. Fundamental passband modes}

In the five cell SPL cavities, the four additional modes of the fundamental passband can drive beam instabilities, because their $(R / Q)_{n}(\beta)$ values can be significant at certain particle velocities as shown in Fig. 20. The mode frequencies and the $(R / Q)_{n}(\beta)$ obtained with SUPERFISH are listed in Table VIII.

The $\mathrm{TM}_{010,4 / 5 \pi}$ modes, which have the highest $(R / Q)_{n}(\beta)$ values, are used in both cavities for further beam dynamics studies. The mode frequency spread is significantly lower for these modes than for other HOMs. In all simulations a frequency spread of $10 \mathrm{kHz}$ is used.

In the first configuration the $\mathrm{TM}_{010,4 / 5 \pi}$ modes are excited by the nominal pulse structure for five different beam currents, as shown in Fig. 21, and 100 linacs are simulated for each step. Above $Q_{\mathrm{ex}}=5 \times 10^{4}$ the average effective longitudinal $\epsilon$ as well as its deviation increases significantly for $\left\langle I_{b}\right\rangle=400 \mathrm{~mA}$ and losses in some simulation runs occur if $Q_{\mathrm{ex}}>5 \times 10^{5}$. The rate of runs with losses increases to $47 \%$ at $Q_{\mathrm{ex}}=10^{7}$. At $\left\langle I_{b}\right\rangle=200 \mathrm{~mA}$ the influence of the $\mathrm{TM}_{010,4 / 5 \pi}$ modes starts to rise above 
$\mathrm{TM}_{010}$ modes $\beta_{\mathrm{g}}=0.65$ cavity

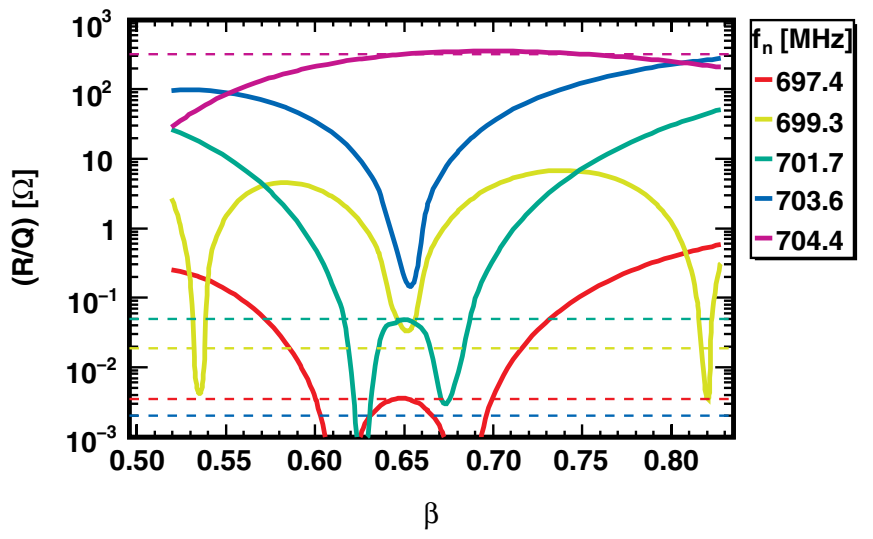

$\mathrm{TM}_{010}$ modes $\beta_{\mathrm{g}}=1$ cavity

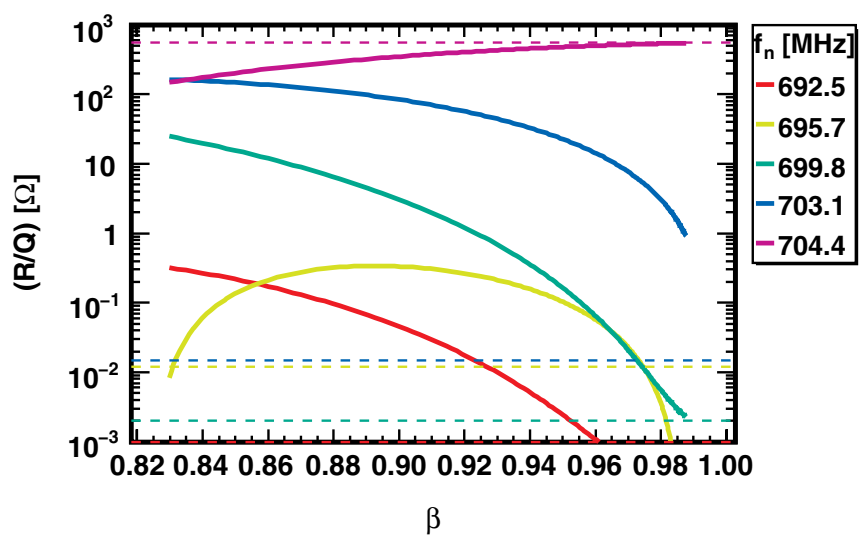

FIG. 20. $(R / Q)_{n}(\beta)$ map of the $\mathrm{TM}_{010}$ modes in the studied cavity. The dashed line is the $(R / Q)_{n}$ value of the mode at the geometrical beta, which can differ several orders of magnitude from the maximum $(R / Q)_{n}$ in the covered velocity range.

$Q_{\mathrm{ex}}=10^{5}$ and no significant effect occur at $\left\langle I_{b}\right\rangle=$ $100 \mathrm{~mA}$ and lower currents. The strong influence of the $\mathrm{TM}_{010,4 / 5 \pi}$ is due to the high $(R / Q)_{n}$ values at the beginning and end of the medium beta section. This effect can only be reduced by reducing the energy range, which is covered by the medium beta cavity.

The $\mathrm{TM}_{010,3 / 5 \pi}$ mode in the high beta cavity and the $\mathrm{TM}_{010,2 / 5 \pi}$ mode in the medium beta cavity are close to a chopping machine line at $699.998 \mathrm{MHz}$ created by a $50 / 80$ chopping pattern. A worst case scenario would be if the mean mode frequency in both cavities is identical with the chopping machine line, which is simulated and shown in Fig. 22. For comparison the simulation is repeated with the mode frequencies obtained from SUPERFISH, see Fig. 23, and with the nominal pulse structure, see Fig. 24. All simulations are done for ten linacs.

If the mean mode frequency falls directly on that chopping machine line, $\epsilon$ increases significantly. At $400 \mathrm{~mA}$ the beam exceeds the rf growth limit above $Q_{\mathrm{ex}}=5 \times 10^{4}$, where also losses occur and operation would only be possible, if the mode is strongly damped. At nominal

TABLE VIII. $\quad \mathrm{TM}_{010}$ passband modes-SUPERFISH results.

\begin{tabular}{lcccc}
\hline \hline Cavity & \multicolumn{2}{c}{$\beta_{g}=0.65$} & \multicolumn{2}{c}{$\beta_{g}=1.0$} \\
\hline Mode & $\begin{array}{c}f_{n} \\
{[\mathrm{MHz}]}\end{array}$ & $\begin{array}{c}(R / Q)_{n}\left(\beta_{\mathrm{g}}\right) \\
{[\Omega]^{\mathrm{a}}}\end{array}$ & $\begin{array}{c}f_{n} \\
{[\mathrm{MHz}]}\end{array}$ & $\begin{array}{c}(R / Q)_{n}\left(\beta_{\mathrm{g}}\right) \\
{[\Omega]^{\mathrm{a}}}\end{array}$ \\
\hline $\mathrm{TM}_{010}, 0$ & 697.4 & 0.003 & 692.6 & 0.001 \\
$\mathrm{TM}_{010}, 2 / 5 \pi$ & 699.3 & 0.019 & 695.7 & 0.012 \\
$\mathrm{TM}_{010}, 3 / 5 \pi$ & 701.7 & 0.049 & 699.8 & 0.002 \\
$\mathrm{TM}_{010}, 4 / 5 \pi$ & 703.7 & 0.002 & 703.1 & 0.015 \\
$\mathrm{TM}_{010}, \pi$ & 704.4 & 318.773 & 704.4 & 561.928 \\
\hline \hline
\end{tabular}

${ }^{\mathrm{a}}$ Linac definition.

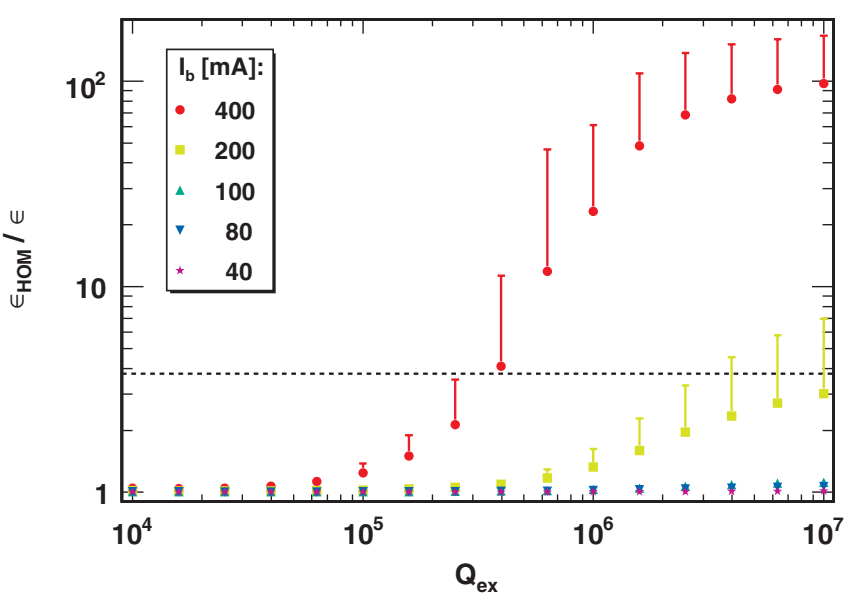

FIG. 21. Average longitudinal phase space increase and upper RMS error against the damping $Q_{\text {ex }}$ caused by the $\mathrm{TM}_{010,4 / 5 \pi}$ modes and different currents. Above the rf limit (dashed line) lossy runs occur. Settings: $\mathrm{TM}_{010,4 / 5 \pi}$ mode, $\sigma_{f_{n}}=10 \mathrm{kHz}$, $N=100$, see Table VI.

beam current the beam stays stable, but $\epsilon$ starts to grow above $Q_{\mathrm{ex}}=10^{4}$. All simulations away from the resonance show no significant longitudinal phase space increase even when using 10 times the nominal current. A distance of a few $100 \mathrm{kHz}$ to the chopping machine line is sufficient to avoid resonance excitation and a significant longitudinal phase space increase.

In [49] the damping, provided by the fundamental power coupler, is estimated for the fundamental passband modes. The damping is sufficient for all scenarios as long as the beam current does not exceed $100 \mathrm{~mA}$. At $400 \mathrm{~mA} \epsilon$ exceeds the rf growth limit at the expected $Q_{\mathrm{ex}}$ in the worst case chopping scenario and for the excitation of the $\mathrm{TM}_{0104 / 5 \pi}$ mode. 


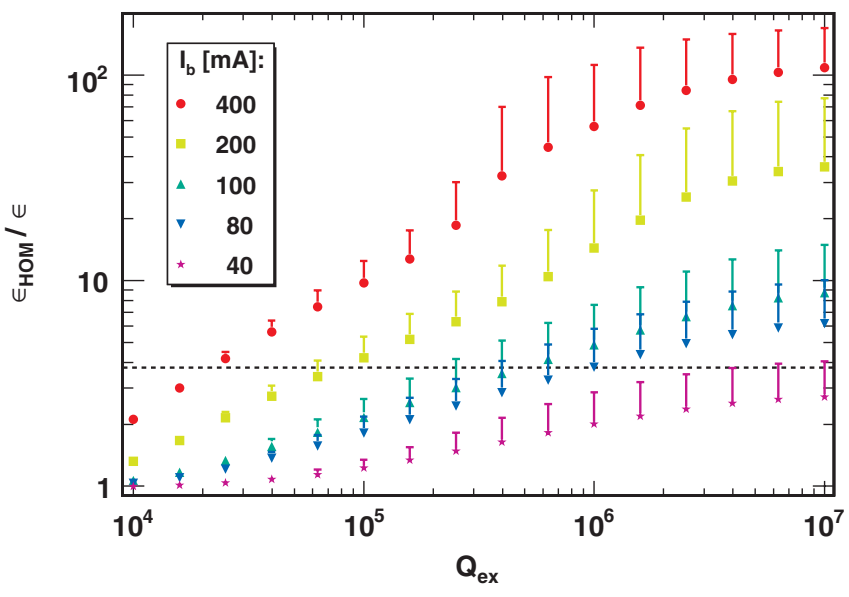

FIG. 22. Average longitudinal phase space increase and upper RMS error against the damping $Q_{\text {ex }}$ caused by the $\mathrm{TM}_{010,3 / 5 \pi}$ mode and $\mathrm{TM}_{010,2 / 5 \pi}$ mode and a chopped beam, where the mode frequency is shifted to the chopping machine line. The phase space increase due to rf errors is indicated with the dashed line. Settings: passband modes, $\left\langle f_{n}\right\rangle=699.998 \mathrm{MHz}, \sigma_{f_{n}}=$ $10 \mathrm{kHz}$, chopping pattern: 50/80, $N=10$, see Table VI.

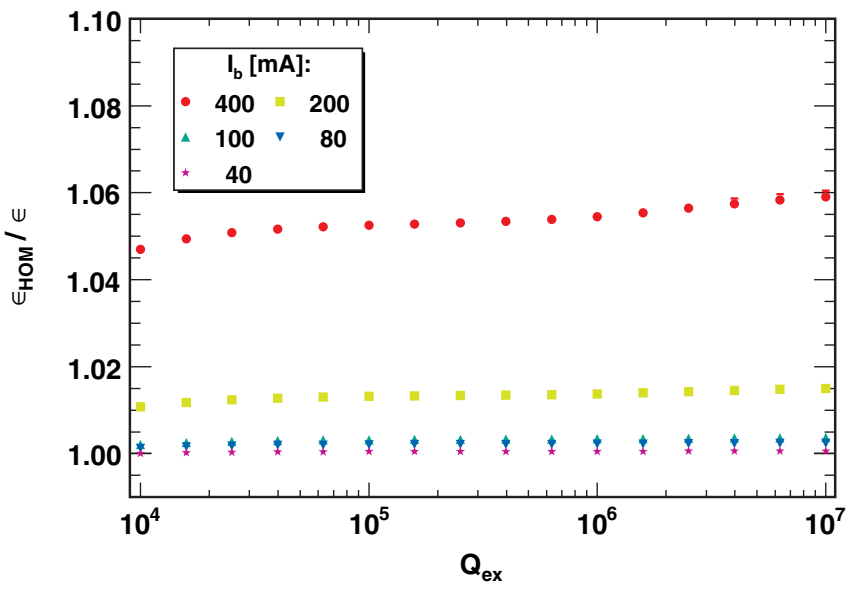

FIG. 23. Average longitudinal phase space increase and RMS error against the damping $Q_{\mathrm{ex}}$ caused by the $\mathrm{TM}_{010,3 / 5 \pi}$ mode and $\mathrm{TM}_{010,2 / 5 \pi}$ mode and a chopped beam. Settings: passband modes, $\sigma_{f_{\text {ном }}}=10 \mathrm{kHz}$, chopping pattern: $50 / 80, N=10$, see Table VI.

\section{TRANSVERSE PLANE}

In contrast to the longitudinal plane a HOM that affects the transverse plane can only be exited by an off-axis beam, because $E_{z}(0, \phi, z)$ is zero for all these modes. First, the parameters and assumptions needed to execute simulations in the transverse plane are summarized. Then effects such as beam break up threshold current, beam noise, alignment errors, and substructured pulses are investigated in detail.

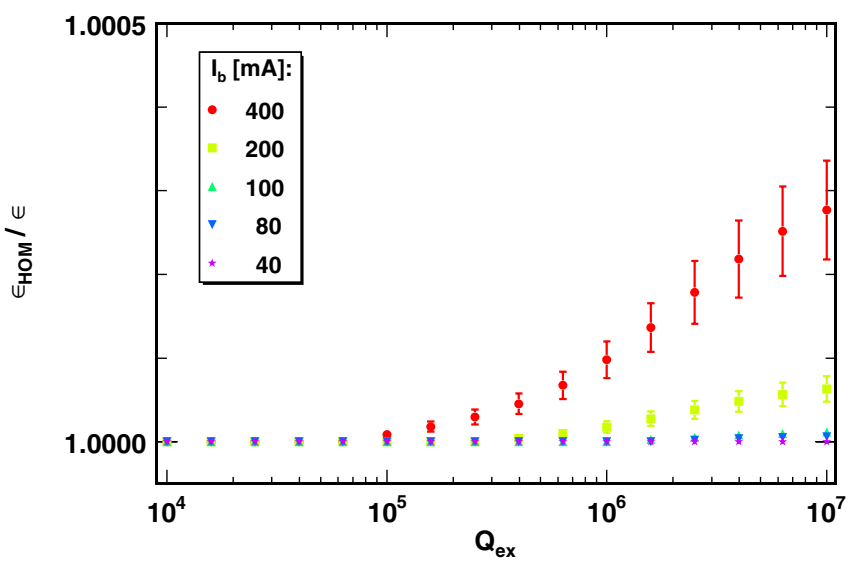

FIG. 24. Average longitudinal phase space increase against the damping $Q_{\text {ex }}$ caused by the $\mathrm{TM}_{010,3 / 5 \pi}$ mode and $\mathrm{TM}_{010,2 / 5 \pi}$ mode. Settings: passband modes, $\sigma_{f_{n}}=10 \mathrm{kHz}, N=10$, see Table VI.

\section{A. Simulation input parameters}

It is assumed for the SPL that the beam enters the linac on axis with a Gaussian position and momentum spread listed in Table IX together with all other default simulation settings used in the transverse plane. An explicit study of off-axis injected beams is done as well and will be discussed later in this section.

A bunch is defined as lost if the transverse displacement at the end of a section is larger than the iris radius of the cavity, which is a very optimistic value. The aim of this study is not to monitor losses, but to limit the maximum

TABLE IX. Default settings used in all transversal beam dynamics simulations.

\begin{tabular}{lccc}
\hline \hline Injected beam & & Mean & $\sigma$ \\
\hline$E_{\text {input }}$ & {$[\mathrm{MeV}]$} & 160 & 0.0 \\
$\phi$ & {$[$ deg at $704 \mathrm{MHz}]$} & -15 & 0.0 \\
$x$ & {$[\mathrm{~mm}]$} & 0.0 & 0.3 \\
$x^{\prime}$ & {$[\mathrm{mrad}]$} & 0.0 & 0.3 \\
$I_{\text {beam }}$ & {$[\mathrm{mA}]$} & 400 & $3 \%$ \\
\hline Pulse structure & & & \\
\hline$T_{b}$ & {$[\mathrm{ps}]$} & 2.84 & \\
$T_{p}$ & {$[\mathrm{~ms}]$} & 1.00 & \\
$T_{r}$ & {$[\mathrm{~ms}]$} & 20.0 & \\
\hline Dipole mode $\beta_{g}=0.65 \mathrm{cavity}$ & & \\
\hline$f_{n}$ & {$[\mathrm{MHz}]$} & 1020 & 1 \\
$(R / Q)_{\perp, n}(\beta)$ & {$[\Omega]$} & $8 \times 10^{-3}-103$ & \\
$Q_{\mathrm{ex}}$ & & $10^{7}$ & \\
\hline Dipole mode $\beta_{g}=1.0 \mathrm{cavity}$ & & \\
\hline$f_{n}$ & {$[\mathrm{MHz}]$} & 915 & 1 \\
$(R / Q)_{\perp, n}(\beta)$ & {$[\Omega]$} & $38-57$ & \\
$Q_{\mathrm{ex}}$ & & $10^{7}$ & \\
\hline \hline
\end{tabular}


induced voltage by an off-axis bunch. For precise loss studies the beam halo has to be modeled as well as the exact linac layout including magnet and alignment errors.

\section{B. Initial simulation}

Before looking at the impact of dipole modes, a simulation only with injection beam noise, including charge jitter, transverse displacement, and tilt, is carried out and used as reference. The resulting phase space distribution at the end of the linac is shown in Fig. 25. Repeating the simulations with one dipole mode present in each cavity, with a HOM frequency spread of $1 \mathrm{MHz}$, a beam current of $400 \mathrm{~mA}$, and a $Q_{\mathrm{ex}}=10^{7}$, leads only to modest changes in the phase space distribution, as illustrated in Fig. 26.

As in the longitudinal plane an effective transverse phase space area, created by one pulse and calculated by

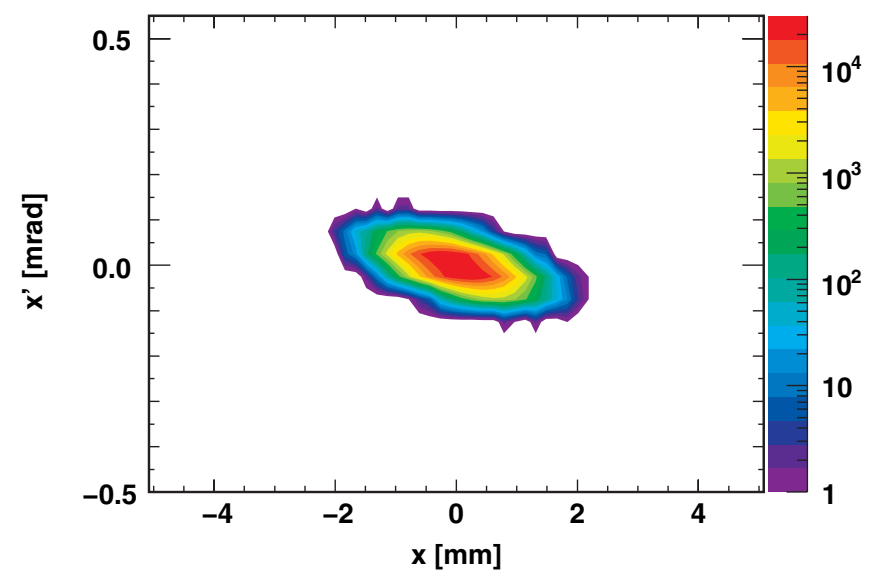

FIG. 25. 2D phase space plot of the second pulse at the exit of the linac without dipole modes present. This distribution is used as a reference for subsequent studies. Settings: no $\mathrm{HOM}, N=1$, see Table IX.

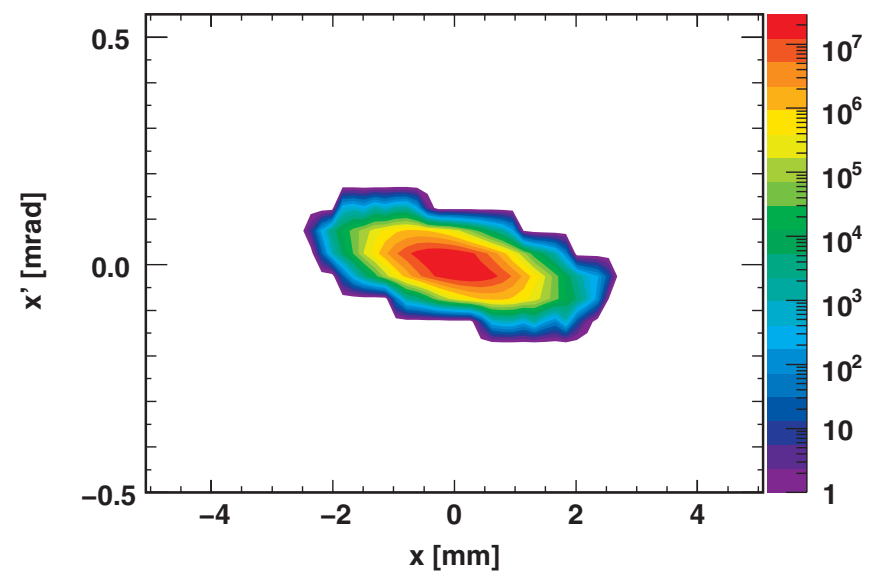

FIG. 26. 2D phase space plot of the second pulse at the exit of the linac with one dipole mode present for 1000 linacs. Settings: $N=1000$, see Table IX.

$$
\epsilon=\pi \sqrt{\left\langle x^{2}\right\rangle\left\langle x^{\prime 2}\right\rangle-\left\langle x x^{\prime}\right\rangle^{2}}
$$

can be used. By normalizing it with a reference area, which is the effective phase space area without HOM impact, it can be used as a measure for the HOM influence.

The average and maximum HOM voltage along the linac after the second pulse for 1000 different linacs, using the same settings as before, are shown in Fig. 27. The oscillations observed in the average HOM voltage in the high beta section are due to the betatron oscillations along the linac. Looking at the maximum HOM voltage, an increase along the high beta section is observed while the $R / Q_{\perp}(\beta)$ decreases. This result is consistent with analytical studies like [5].

\section{Current and damping sweeps}

As in the longitudinal plane, the upper limit for $Q_{\mathrm{ex}}$ is set to $10^{8}$, which is the damping level, where the influence of pulse-to-pulse coupling starts to rise.

The beam current $\left\langle I_{b}\right\rangle$ is varied between the nominal current up to $400 \mathrm{~mA}$. 100 different linacs are analyzed for each combination of $Q_{\mathrm{ex}}$ and $\left\langle I_{b}\right\rangle$ and the resulting average transverse phase space increase and its variance is illustrated in Fig. 28.

At a $Q_{\text {ex }}$ of $10^{4}$, no growth is observed for all currents. In case of $\left\langle I_{b}\right\rangle=400 \mathrm{~mA}$, the influence starts to rise at $Q_{\mathrm{ex}}=10^{5}$ and reaches a plateau level around $Q_{\mathrm{ex}}=10^{7}$ before starting to rise further above $Q_{\mathrm{ex}}=4 \times 10^{7}$ due to pulse-to-pulse coupling. Also the spread in the single simulation results increases significantly. At nominal current below $Q_{\mathrm{ex}}=4 \times 10^{7}$ no effect is visible. In general, the phase space increase is quadratic with the beam current and the dependency on $Q_{\mathrm{ex}}$ is the same as in the longitudinal plane.

Based on these results all further studies are carried out at a $Q_{\mathrm{ex}}=10^{7}$ and $\left\langle I_{b}\right\rangle=400 \mathrm{~mA}$. At this damping level

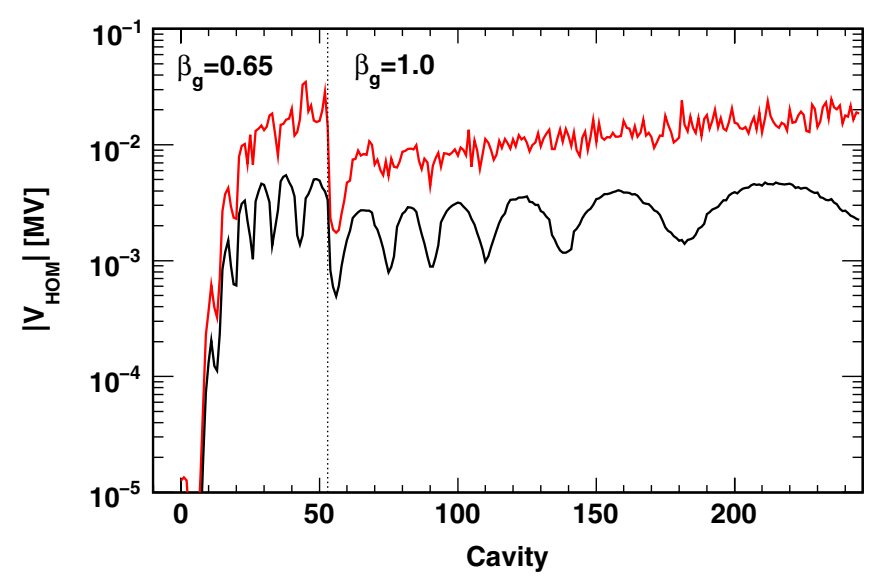

FIG. 27. Average (black) and maximum (red) transverse HOM voltage present in the cavities after two pulses for 1000 linacs. Settings: $N=1000$, see Table IX. 


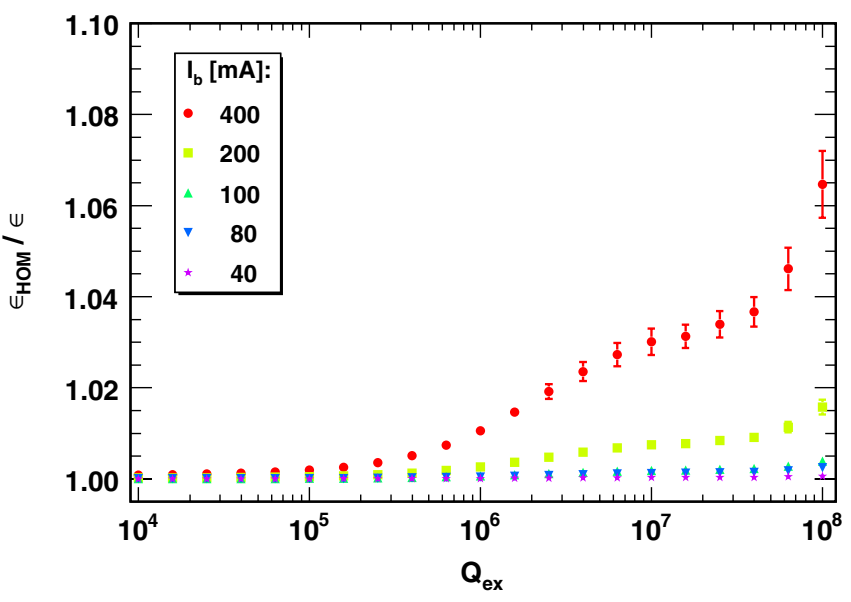

FIG. 28. Average transverse phase space increase of the beam against the damping $Q_{\mathrm{ex}}$ and different beam currents $\left\langle I_{b}\right\rangle$ where one HOM per cavity is present for 100 linacs. The influence of the HOM rises above $Q_{\mathrm{ex}}=10^{5}$ and is proportional to $\left\langle I_{b}\right\rangle^{2}$. The plateau around $Q_{\mathrm{ex}}=10^{7}$ is due to the pulse period structure. Settings: $N=100$, see Table IX.

the pulses are still independent from each other and the HOM voltage history does not have to be taken into account.

\section{Injection beam noise}

Injection beam noise is essential to excite dipole modes, because the beam on axis has no effect. Following the logic, applied in the longitudinal plane, simulations with different beam noise patterns are executed to define a representative sample, which is used in the subsequent studies. 1000 simulations are carried out in each case, where the following parameter is varied: (i) injection noise pattern; (ii) HOM frequency pattern; (iii) injection noise and HOM frequency pattern.

The deviation in all cases is around $0.3 \%$ and all mean values deviate less than $1 \sigma$. Based on these results, the effect of different injection noise patterns is not considered in further simulations and only the HOM frequency pattern will be varied.

In Fig. 29 the effect of the charge scatter is shown, which is less than in the longitudinal plane. The observed increase in $\epsilon$ is proportional to $\sigma_{\mathrm{q}}^{2}$ and scales also with the beam current in the same manner. Even for a charge scatter of $10 \%$ the phase space increase is the same order of magnitude as the deviation due to different injection noise or HOM frequency patterns.

\section{E. HOM frequency spread}

The HOM frequency spread is important in building up instabilities. Below a certain frequency spread threshold level, the beam position modulation induced by a dipole mode in a cavity is constructively increased in further downstream cavities. In order to find this threshold level,

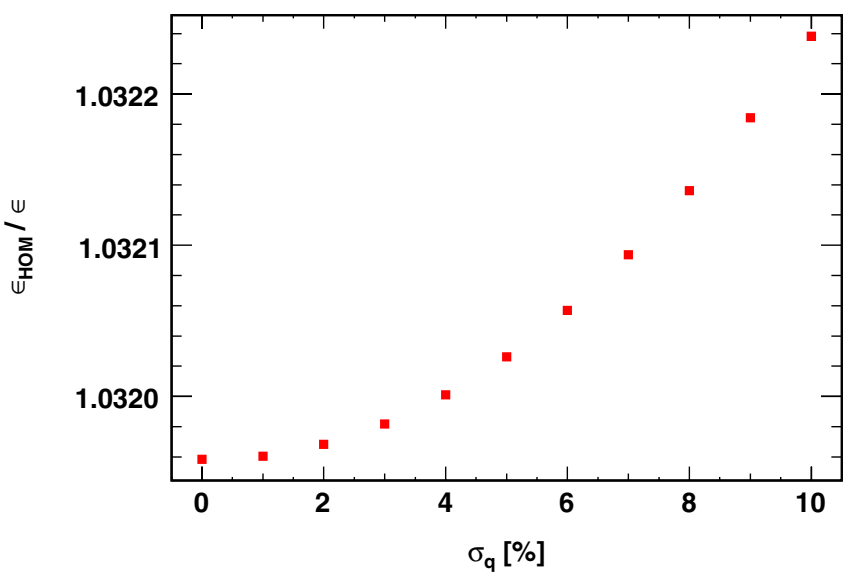

FIG. 29. Average effective transverse phase space growth of the beam as a function of the Gaussian bunch to bunch charge scatter. Settings: $N=100$, see Table IX.

the HOM frequency spread is varied between $10 \mathrm{kHz}$ and $10 \mathrm{MHz}$.

The average and maximum transverse phase space increase for 100 linacs is shown in Fig. 30. No values are plotted for $10 \mathrm{kHz}$ because beam losses occurred there. From the graph, one can see that the threshold level is in the order of some $100 \mathrm{kHz}$, much smaller than the HOM frequency spread in the SPL cavities, which is expected to be at the level of several MHz.

Simulations carried out with the nominal beam current indicate the same threshold level, but the transverse phase space increase is smaller due to the reduced current.

This confirms the analytical work on transverse beam breakup of transient beams in [5], which describes the HOM frequency spread as an additional HOM damping. Repeating the simulation with lower $Q_{\text {ex }}$ values leads to a shift of the threshold value to lower frequency spread values, as expected. A higher HOM frequency spread is

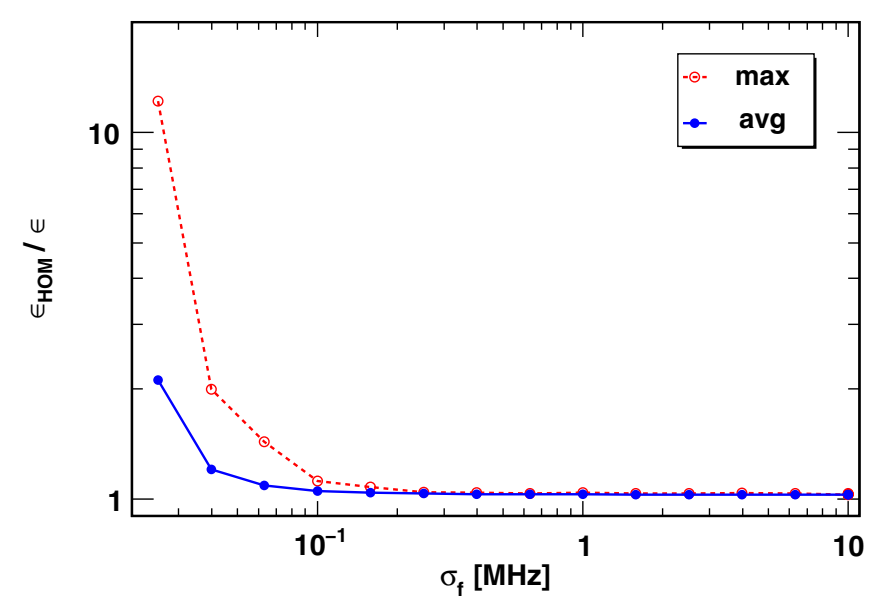

FIG. 30. Average and maximum transverse phase space increase dependency on the HOM frequency spread. Settings: $N=100$, see Table IX. 
TABLE X. Chopping patterns and the used resonance frequencies for dipole modes in both linac sections.

\begin{tabular}{lcc}
\hline \hline Mode & Chopping pattern $(m / N)$ & $f_{(\mathrm{C}) \mathrm{ML}}[\mathrm{MHz}]$ \\
\hline 1 & No chopping & 1056.60 \\
2 & $5 / 8$ & 1012.58 \\
3 & $50 / 80$ & 1016.98 \\
4 & $500 / 800$ & 1018.30 \\
\hline \hline
\end{tabular}

needed for higher $Q_{\mathrm{ex}}$ to sufficiently reduce the HOM excitation and the caused phase space increase.

\section{F. Substructured pulses}

The influence of the pulse substructure is investigated in detail in the transverse plane, too. Therefore, the same chopping patterns are used as in the longitudinal plane, but different resonance frequencies, which are listed in Table X. The integrated charge per pulse is kept constant, which means an increase of the bunch current in case of chopping. The increase in phase space at the exit of the linac for different chopping patterns, off and on resonance, as a function of the damping is shown in Fig. 31. For each configuration 100 linacs with different HOM frequency patterns are simulated.

Introducing chopping leads to an increase in $\epsilon$ due to the increased bunch charge. This can be validated by repeating the simulation with increased beam current but without chopping. Shifting the HOM frequency to a chopping machine line causes a further increase in the phase space area. In this context it should be stated that the induced transverse HOM voltage scales with $\omega_{n}^{2}$. A chance of the HOM frequency leads to a change in the transverse phase space increase, which is roughly proportional to $\omega_{n}$.

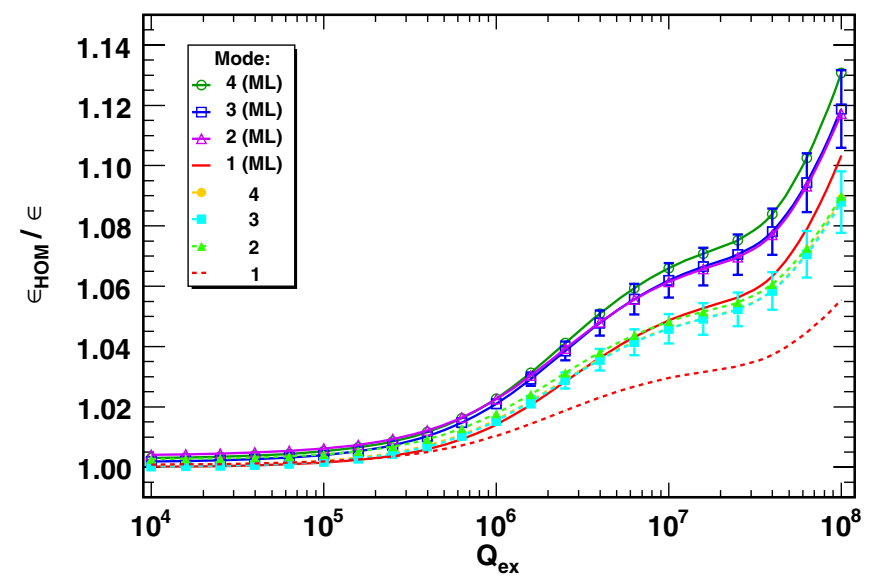

FIG. 31. Average transverse phase space increase against the damping $Q_{\mathrm{ex}}$ and for different chopping patterns on (ML) and off a resonance. The error bars are only plotted for the 50/80 chopping pattern and about the same for the other cases. Settings: chopping, $\left\langle f_{\mathrm{HOM}}\right\rangle$ partly shifted (see Table $\mathrm{X}$ ), $N=100$, see Table IX.
Hence, the observed phase space increase is only partly due to the resonance condition.

The characteristic $Q_{\mathrm{ex}}$ dependency is the same as in the nominal case. The difference between the different chopping patterns is relatively small and the mean values are all inside an error of one sigma.

In general, the effect of chopping in the transverse plane is far less dramatic than in the longitudinal plane and has the same effect as shifting a dipole mode to a machine line. At 10 times the nominal current the phase space increase is less than $10 \%$, even for $Q_{\mathrm{ex}}=10^{7}$.

\section{G. Alignment errors}

The effects of displaced beams and cavities can drive dipole modes, as the beam passes the cavities off axis. There is no additional first order effect on the monopole modes, because a longitudinally displaced cavity causes the same effect as a phase error, which can be treated as a klystron error. Hence, alignment errors are only treated in the transverse plane in this survey. First, the cavities are displaced inside the cryomodule and second the effect of off-axis injection is analyzed.

\section{Cavity alignment}

The cavities are randomly displaced transversely around the beam axis. A Gaussian distribution with a deviation of $\sigma_{d x}$ is considered. The magnets are assumed not to be affected. Additional transverse rf kicks, introduced by the accelerating mode in a displaced cavity, are also not considered in this simulation [52]. The $\sigma_{d x}$ is varied in the range from 0.1 up to $10 \mathrm{~mm}$ and for each $\sigma_{d x} 100$ linacs are simulated, where only the cavity displacement is varied. All other parameters are the default ones. Figure 32 shows the phase space increase at the end of the linac. The

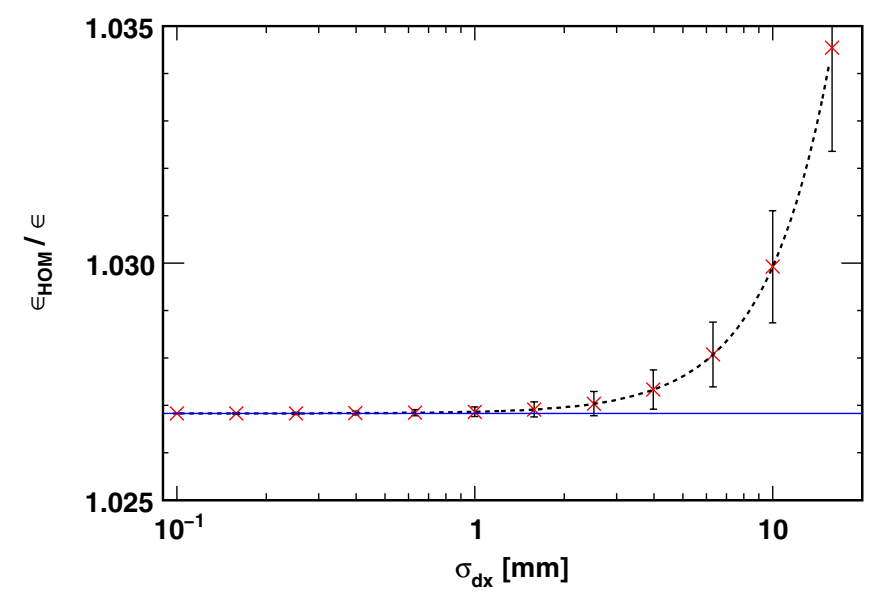

FIG. 32. Average transverse phase space increase against cavity alignment errors (red crosses with error bars). A second order polynomial is fitted through the data (dashed line). For comparison the phase space increase without cavity displacement (blue line) is added. Settings: $N=100$, see Table IX. 
average increase in $\epsilon$ is proportional to $\sigma_{d x}^{2}$ and starts to rise above $\sigma_{d x}=1 \mathrm{~mm}$ compared to the simulation result without cavity displacement. This result agrees with the analytic predictions in [53]. For smaller displacements no significant phase space increase is observed. Even for $\sigma_{d x}=10 \mathrm{~mm}$ and $400 \mathrm{~mA}$ the additional phase space increase is only about $3 \%$ and in the same order of magnitude as the variation due to different HOM frequency patterns.

From experience at other labs, it is expected that the cavities can be aligned with a precision in the order of $1 \mathrm{~mm}$. At that level, dipole modes do not affect the beam more than in the case of a perfect cryomodule. So cavity alignment errors are no concern for HOM excitation in the SPL, within the simulation limitations. The influence of magnet displacement errors has been studied for different focusing schemes in [54].

\section{Off-axis injection}

The beam is injected off axis into the linac. All further downstream cryomodules are assumed to be perfectly aligned with the first one. $\epsilon$ is not translation invariant and a mean off-axis extraction position leads to an increase of $\epsilon$.

To get rid of this effect, the following translation invariant formula is used to calculate the phase space area in this subsection:

$$
\epsilon^{*}=\pi \sqrt{\left\langle x^{2}\right\rangle\left\langle x^{\prime 2}\right\rangle-\left\langle x x^{\prime}\right\rangle^{2}-2 \bar{x} \cdot \bar{x}^{\prime}+\bar{x}^{\prime 2}\left\langle x^{2}\right\rangle+\bar{x}^{2}\left\langle x^{\prime 2}\right\rangle},
$$

where $\bar{x}$ and $\bar{x}^{\prime}$ are the mean values of the position and momentum distribution. Using (28) instead of (27), the phase space increase is then only due to the effect of the dipole mode and shown in Fig. 33. There the phase space

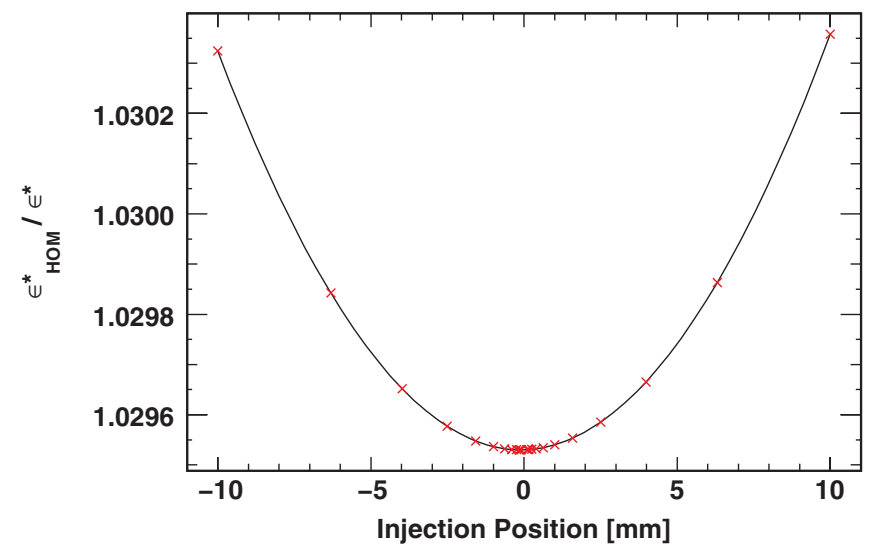

FIG. 33. Average $\epsilon^{*}$ growth in case of an off-axis injected beam with a HOM present and normalized with same off-axis beam without HOMs. The phase space increases with the injection offset, but is fare less than the deviation $\sigma\left(\epsilon^{*}\right)=0.28 \%$ introduced by different HOM frequency patterns. Settings: $N=100$, see Table IX.

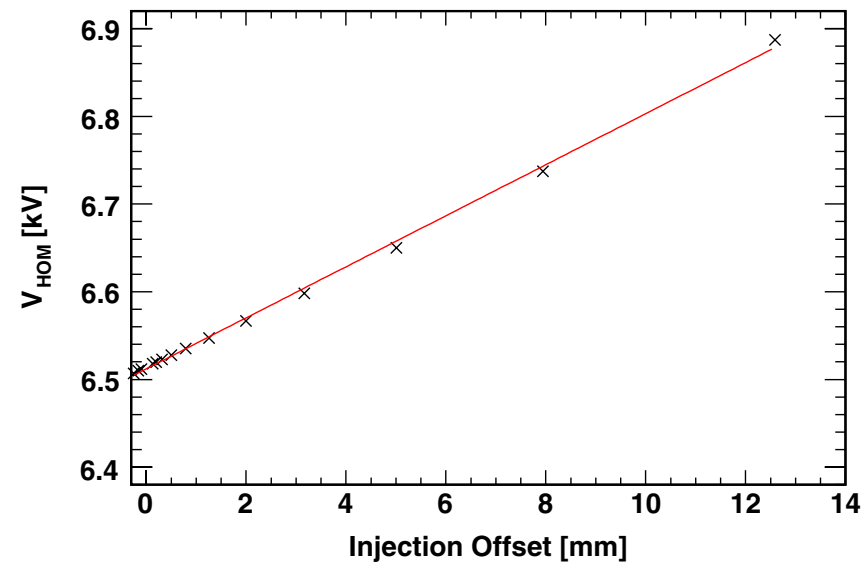

FIG. 34. Maximum observed HOM voltage in the high beta section as function of the injection error and a linear fit through the data. Settings: $N=100$, see Table IX.

increases with the injection offset approximately quadratically. The mean extraction position as well as the extraction declination increases linear with the injection offset, which is also reflected in the HOM voltage, shown in Fig. 34, because the bunch induced voltage in a dipole mode is proportional to the off-axis position $x$. This result is in good agreement with [55], where the effect of off-axis injection is discussed analytically.

In case of off-axis injection the influence of the dipole mode increases, but not significantly. The additional increase is less than $1 \%$ in case of injection $10 \mathrm{~mm}$ away from the beam axis and a beam current of $400 \mathrm{~mA}$. The deviation in the phase space area due to different HOM frequency patterns is $2.8 \%$ and so much higher than the influence of an off-axis injected beam. Hence, alignment seems to be no major issue in the context of HOMs for the SPL. Further multiparticle simulations, however, have to be carried out to study the general beam sensitivity with respect to alignment errors of transverse and longitudinal focusing.

\section{CONCLUSION}

A new code SMD, dedicated to simulate the influence of higher order modes in superconducting proton linacs, is developed, benchmarked, and used to analyze the potential risk of HOMs in the SPL. Simulations are carried out in the longitudinal and transverse plane.

One of the results is that dipole modes do not affect a proton beam significantly as it is the case for electrons. In the longitudinal plane the influence of injection beam noise, HOM frequency spread, rf errors, machine lines, and substructured pulses are investigated in detail as a function of the beam current and the HOM damping. Using the SPL design parameters and estimated errors, only resonance lines are critical for a stable operation. The principal machine lines are known and can be taken into account in the cavity design, so that no HOM meets 
such a resonance. However, machine lines created by the pulse substructure depend on the chopping pattern and can occur at any frequency. A strong HOM damping of $Q_{\mathrm{ex}}=$ $10^{5}$ is recommended to allow any possible pulse substructure. In the absence of any pulse substructure only a moderate damping in the order of $Q_{\mathrm{ex}}=10^{7}$ is needed. In the transverse plane the influence of beam injection noise, HOM frequency spread, resonances, and cavity alignment is investigated in detail and no instabilities have been found at a moderate HOM damping level of $Q_{\text {ex }}=10^{7}$.

The variety in the pulse substructure is the main difference of the SPL to other high power superconducting proton linacs such as SNS or ESS and causes stricter HOM damping requirements in the order of $Q_{\mathrm{ex}}=10^{5}$ as compared, for example, to SNS. In Project X also high frequently chopping patterns are foreseen, but there the average beam current is a factor 40 smaller than in the SPL. Hence, resonant HOM excitation is less critical there.

\section{APPENDIX A: CAVITY SHAPE PARAMETERS}

The cavity shape parameters are illustrated in Fig. 35 and the used values are listed in Table XI.

\section{B. ENERGY VERSUS MOMENTUM GAIN}

We will show in the following that the same (small) momentum change, once in longitudinal, once in transverse direction, corresponds to very different energy gain, much lower in the transverse case. This relation holds for any particle speed from classical $v \ll c$ to highly relativistic $v \approx c$ cases.

To include any particle speed we use the always precise relativistic energy-momentum relation,

$$
E_{0}=\sqrt{(c \cdot \vec{p})^{2}+\left(m_{0} c^{2}\right)^{2}}=\sqrt{c^{2} \cdot\left(p_{\|}^{2}+p_{\perp}^{2}\right)+\left(m_{0} c^{2}\right)^{2}} .
$$

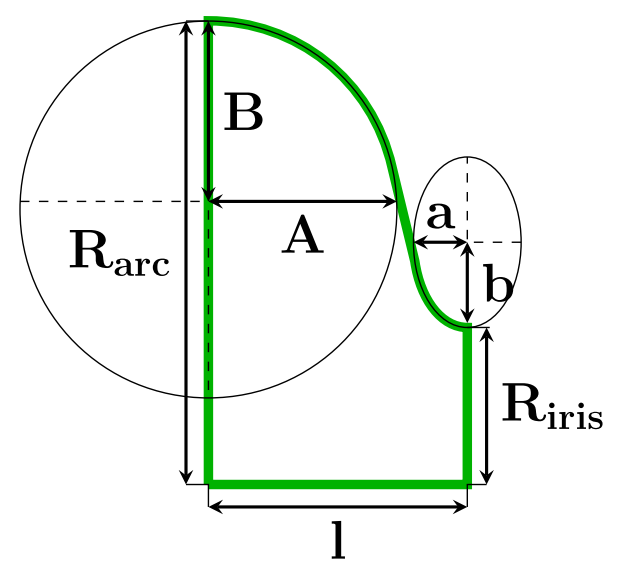

FIG. 35. Cavity with labels of the shape parameters used in Table XI.
TABLE XI. Shape parameters of the medium and high beta cavities. Both are symmetric five cell elliptical SC cavities. All values are given in $\mathrm{mm}$.

\begin{tabular}{lcrrr}
\hline \hline Cavity & \multicolumn{2}{c}{$\beta_{g}=0.658$} & \multicolumn{2}{c}{$\beta_{g}=1$} \\
\hline Cell & mid & end & mid & end \\
$R_{\text {arc }}$ & 186.4 & 186.4 & 190.8 & 190.8 \\
$R_{\text {iris }}$ & 45.0 & 45.0 & 64.6 & 70.0 \\
$l_{\text {cell }}$ & 70.0 & 70.0 & 106.5 & 103.0 \\
$A$ & 45.1 & 45.1 & 77.5 & 76.9 \\
$B$ & 45.1 & 49.6 & 77.5 & 74.5 \\
$a$ & 12.1 & 12.1 & 22.1 & 18.5 \\
$b$ & 15.8 & 15.7 & 35.1 & 24.9 \\
\hline \hline
\end{tabular}

The coordinate system for the following considerations is chosen such that before deflection $p_{\perp}=0$.

For the longitudinal case we let an accelerating cavity induce a longitudinal momentum increase $\Delta p_{\|}$, very small compared to $p_{\|}$. Then the corresponding energy change of the particle is

$$
\begin{aligned}
& \Delta E\left(\Delta p_{\|}\right)=\sqrt{c^{2} \cdot\left(p_{\|}+\Delta p_{\|}\right)^{2}+\left(m_{0} c^{2}\right)^{2}}-E_{0} \text { or } \\
& \Delta E\left(\Delta p_{\|}\right)=E_{0}\left(\sqrt{1+c^{2} \frac{2 p_{\|} \cdot \Delta p_{\|}+\Delta p_{\|}^{2}}{E_{0}^{2}}}-1\right) .
\end{aligned}
$$

The fraction under the root is much smaller than 1 for any particle speed. In the classical case $E_{0}^{2}$ is about the square of the rest energy $E_{0} \approx m_{0} c^{2}$, and the fraction becomes about $2 v_{\|} \Delta v_{\|} / c^{2} \ll 1$. In the relativistic case with $E_{0} \gg$ $m_{0} c^{2}$ we have from (B1) $c p_{\|} / E_{0} \rightarrow 1$; hence, the fraction tends against $2 \Delta p_{\|} / p_{\|} \ll 1$. Then we get to first order

$$
\Delta E\left(\Delta p_{\|}\right) \approx \frac{c^{2}}{E_{0}} p_{\|} \Delta p_{\|}
$$

For the transverse case we let a deflecting cavity induce a transverse momentum kick $\Delta p_{\perp}$ and again we can reasonably assume that $\Delta p_{\perp} \ll p_{\|}$. In assuming the unproven hypothesis that $p_{\|}$is unchanged when passing the purely deflecting cavity - see the core of this paper-we can determine the energy change to be

$$
\begin{gathered}
\Delta E\left(\Delta p_{\perp}\right)=\sqrt{c^{2} \cdot\left(p_{\|}^{2}+\Delta p_{\perp}^{2}\right)+\left(m_{0} c^{2}\right)^{2}}-E_{0} \text { or } \\
\Delta E\left(\Delta p_{\perp}\right)=E_{0}\left(\sqrt{1+c^{2} \frac{\Delta p_{\perp}^{2}}{E_{0}^{2}}}-1\right) .
\end{gathered}
$$

Also here the fraction under the root is very small compared to 1 for any particle speed; hence, we get to first order 


$$
\Delta E\left(\Delta p_{\perp}\right) \approx \frac{c^{2}}{E_{0}} \frac{\Delta p_{\perp}^{2}}{2} .
$$

From this we get the ratio $r$ of energy change for a transverse and a longitudinal momentum change

$$
r=\frac{\Delta E\left(\Delta p_{\perp}\right)}{\Delta E\left(\Delta p_{\|}\right)}=\frac{\Delta p_{\perp}}{2 p_{\|}} \cdot \frac{\Delta p_{\perp}}{\Delta p_{\|}} .
$$

For a realistic deflection $\Delta p_{\perp} / p_{\|}$is a very small number. This means that for the same (small) momentum change the energy change in the transverse case is considerably smaller than in the longitudinal case.

All these considerations are only based on kinematics and do not consider how the deflection was realized, by either electric or magnetic fields. For the latter case it is known that the energy change is even rigorously zero, i.e., due to the curvature of the track the momentum in the initial forward direction diminishes just by the right amount.

[1] F. Gerigk, M. Baylac, E. B. Mora, F. Caspers, S. Chel, J. M. Deconto, R. Duperrier, E. Froidefond, R. Garoby, K. Hanke, C. Hill, M. Hori, J. Inigo-Goln, K. Kahle, T. Kroyer, D. Kuechler, J.-B. Lallement, M. Lindroos, A. M. Lombardi, A. Hernndez, M. Magistris, T. K. Meinschad, A. Millich, E. N. Messomo, C. Pagani, V. Palladino, M. Paoluzzi, M. Pasini, P. Pierini, C. Rossi, J. P. Royer, M. Sanmarti, E. Sargsyan, R. Scrivens, M. Silari, T. Steiner, J. Tückmantel, D. Uriot, and M. Vretenar, Conceptual Design of the SPL II: A high-power superconducting $H^{-}$linac at CERN, edited by F. Gerigk, Yellow Reports No. CERN2006006 (CERN, Geneva, 2006) p. 104.

[2] O. Brunner, S. Calatroni, E. Ciapala, M. Eshraqi, R. Garoby, F. Gerigk, A. Lombardi, R. Losito, V. Parma, C. Rossi, J. Tuckmantel, M. Vretenar, U. Wagner, and W. Weingarten, Phys. Rev. ST Accel. Beams 12, 070402 (2009).

[3] R. Garoby, in Proceedings of SRF2009, Berlin, Germany (2009), FROBAU04.

[4] "Workshop on cryogenic and vacuum sectorizations of the SPL," (2009). http://indico.cern.ch/event/68499.

[5] C. L. Bohn and J. R. Delayen, Phys. Rev. A 45, 5964 (1992).

[6] R. L. Gluckstern and R. K. Cooper, in Proceedings PAC 1985 [IEEE Trans. Nucl. Sci. 32 (1985)].

[7] D. Jeon, L. Merminga, G. Krafft, B. Yunn, R. Sundelin, J. Delayen, S. Kim, and M. Doleans, Nucl. Instrum. Methods Phys. Res., Sect. A 495, 85 (2002).

[8] J. Tückmantel, Phys. Rev. ST Accel. Beams 13, 011001 (2010).

[9] S.-H. Kim, J. Korean Phys. Soc. 52, 714 (2008).

[10] Y. Zhang, in Proceedings of IPAC'10, Kyoto, Japan (2010), MOZMH01.

[11] M. Eshraqi, M. Brandin, I. Bustinduy, C. Carlile, H. Hahn, M. Lindroos, C. Oyon, S. Peggs, A. Ponton, K. Rathsman, R. Calaga, T. Satogata, and A. Jansson, in Proceedings of IPAC'10, Kyoto, Japan (2010), MOPD053.

[12] M. Lindroos, C. Oyon, and S. Peggs, in Proceedings of SRF2009, Berlin, Germany (2009), FROBAU02.
[13] S. D. Holmes, in Proceedings of IPAC'10, Kyoto, Japan (2010), TUYRA01.

[14] N. Solyak, A. Vostrikov, T. Khabiboulline, A. Saini, and V. Yakovlev, in Proceedings of IPAC'10, Kyoto, Japan (2010), TUPEA020.

[15] Simulate higher order Mode Dynamics (SMD) code repository, http://svnweb.cern.ch/world/wsvn/splhombbu.

[16] H. Padamsee, J. Knobloch, and T. Hays, $R F$ Superconductivity for Accelerators (Wiley-VCH, Berlin, Germany, 2008), p. 521, 2nd ed.

[17] T. P. Wangler, Principles of RF Linear Acceerators, edited by T.P. Wangler (Wiley-VCH, Berlin, Germany, 2008), 2nd ed..

[18] S.-H. Kim, M. Doleans, D. Jeon, and R. Sundelin, Nucl. Instrum. Methods Phys. Res., Sect. A 492, 1 (2002).

[19] RMS bunch length is $2.2 \mathrm{deg}$ at $352 \mathrm{MHz}$ at the end of Linac4.

[20] Throughout this paper the linac definition is used. To convert into the circuit definition the values has to be divided by a factor 2 .

[21] Since the transit time of the bunch is several orders of magnitude faster than the decay time of the mode. It can be assumed for simplification that the cavity beam interaction take place instantaneously at the cavity midplane.

[22] W. K. H. Panofsky and W. A. Wenzel, Rev. Sci. Instrum. 27, 967 (1956).

[23] J. Tückmantel, CERN Technical Report No. CERN-ATSNote-2011-002, 2011.

[24] P. Wilson, in AIP Conference Proceedings (AIP, New York, 1981), Vol. 87.

[25] R. Brun and F. Rademakers, in Proceedings AIHENP'96 Workshop, Lausanne, 1996 [Nucl. Instrum. Methods Phys. Res., Sect. A 389, 81-86 (1997)].

[26] This is not included in codes such as for example TALOBBU or the code used in [8].

[27] M. Matsumoto and T. Nishimura, ACM Trans. Model. Comput. Simul. 8, 3 (1998).

[28] A constant acceleration voltage along the linac is assumed; hence, an energy error cause no acceleration kick error. By including this effect the results would deviate, since this effect is only modeled in SMD.

[29] M. Schuh, J. Tückmantel, J.-L. Biarrotte, and D. Kaltchev, CERN, Technical Report No. sLHC-Project-Note-0010, 2010.

[30] A. Lombardi, S.-H. Kim, and W. Weingarten, CERN Report No. sLHC-Project-Note 0003, 2009.

[31] F. Gerigk, L. Arnaudon, P. Baudrenghien, M. Baylac, G. Bellodi, Y. Body, J. Borburgh, P. Bourquin, J. Broere, O. Brunner, L. Bruno, C. Carli, F. Caspers, S. M. Cousineau, Y. Cuvet, C. De Almeida Martins, T. Dobers, T. Fowler, R. Garoby, B. Goddard, K. Hanke, M. Hori, M. Jones, K. Kahle, W. Kalbreier, T. Kroyer, D. Kchler, A. M. Lombardi, L. A. L'opez-Hernandez, M. Magistris, M. Martini, S. Maury, E. Page, M. Paoluzzi, M. Pasini, U. Raich, C. Rossi, J. P. Royer, E. Sargsyan, J. Serrano, R. Scrivens, M. Silari, M. Timmins, W. Venturini-Delsolaro, M. Vretenar, R. Wegner, W. Weterings, and T. Zickler, Linac4 Technical Design Report, Technical Design Report CERN Report No. CERN-AB-2006-084, 2006.

[32] M. Vretenar, in Proceedings of the LINAC'10, Tsukuba, Japan (2010), WE103. 
[33] J.-L. Biarrotte, Ph.D. thesis, l'université Paris XI Orsay, Université de Paris-Sud U.F.R. Scientifique d'Orsay, 2000.

[34] B. Aune, R. Bandelmann, D. Bloess, B. Bonin, A. Bosotti, M. Champion, C. Crawford, G. Deppe, B. Dwersteg, D. A. Edwards, H. T. Edwards, M. Ferrario, M. Fouaidy, P.-D. Gall, A. Gamp, A. Gössel, J. Graber, D. Hubert, M. Hüning, M. Juillard, T. Junquera, H. Kaiser, G. Kreps, M. Kuchnir, R. Lange, M. Leenen, M. Liepe, L. Lilje, A. Matheisen, W.-D. Möller, A. Mosnier, H. Padamsee, C. Pagani, M. Pekeler, H.-B. Peters, O. Peters, D. Proch, K. Rehlich, D. Reschke, H. Safa, T. Schilcher, P. Schmüser, J. Sekutowicz, S. Simrock, W. Singer, M. Tigner, D. Trines, K. Twarowski, G. Weichert, J. Weisend, J. Wojtkiewicz, S. Wolff, and K. Zapfe, Phys. Rev. ST Accel. Beams 3, 092001 (2000).

[35] R. Wanzenberg, DESY Technical Report 2001.

[36] G. Devanz (private communication).

[37] Ansoft Inc., "HFSS," Homepage.

[38] R.-H. Zeng, M. Schuh, F. Gerigk, R. Wegner, W.-M. Pan, G.-W. Wang, and R. Liu, Chinese Phys. C 34, 99 (2010).

[39] The fundamental passband modes $\left(\mathrm{TM}_{010}\right)$ are analyzed separately.

[40] K. Halbach and R. F. Holsinger, Part. Accel. 7, 213 (1976).

[41] J. Sekutowicz (private communication).

[42] F. Marhauser (private communication).

[43] S. Molloy and R. Calaga, in Proceedings of IPAC'10, Kyoto, Japan (2010), WEPEC055.

[44] M. Liepe, G. Stedman, and N. Valles, in Proceedings of the 23rd Particle Accelerator Conference, Vancouver, Canada, 2009 (IEEE, Piscataway, NJ, 2009), TU5PFP048.
[45] G. Bellodi, M. Eshraqi, J.-B. Lallement, S. Lanzone, A. Lombardi, E. Sargsyan, R. Duperrier, and D. Uriot, in Proceedings of LINAC08, Victoria, BC, Canada (2008), MOP086.

[46] This bunch to bunch charge jitter is ten times higher than measured at SNS [30,47].

[47] D. A. Bartkoski, A. V. Aleksandrov, D. E. Anderson, M. Crofford, C. Deibele, S. Henderson, J. Patterson, C. S. III, and A. Webster, in Proceedings of LINAC06, Knoxville, Tennessee (2006), TUP002.

[48] M. Eshraqi (private communication).

[49] M. Schuh, F. Gerigk, J. Tückmantel, and C. P. Welsch, in Proceedings of IPAC'10, Kyoto, Japan (2010), THPE082.

[50] The induced HOM voltage has the opposite sign than the acceleration voltage and HOM frequency is an integer multiple of the bunch frequency, so the HOM acts always as a decelerating kick.

[51] S.-H. Kim, M. Doleans, and R. Sundelin, HOM findings and HOM induced power in the superconducting linac of the intense pulsed proton accelerator, Technical note 10 , Oak Ridge National Laboratory (2001).

[52] Transverse rf focusing is included in the smooth focusing data obtained from general beam dynamics simulations, where a perfect aligned linac is assumed.

[53] J. R. Delayen, Phys. Rev. ST Accel. Beams 7, 074402 (2004).

[54] M. Eshraqi, "Highly segmented SPL as a mixture of Doublet and FODO focusing," 5th SPL collaboration meeting (2010).

[55] J. R. Delayen, Phys. Rev. ST Accel. Beams 6, 084402 (2003). 